\title{
STRUCTURAL CHANGES AND SEEMINGLY UNIDENTIFIED STRUCTURAL EQUATIONS
}

\author{
IN CHOI \\ Hong Kong University of Science and Technology \\ and \\ University of Southern California
}

\begin{abstract}
This paper introduces structural equations that do not satisfy the rank and/or order condition(s) for identification but still are identifiable. These equations are called seemingly unidentified structural equations. The key to the identifiability of these equations is that the right-hand-side endogenous variables undergo structural changes with respect to the exogenous and/or predetermined variables. To estimate the seemingly unidentified structural equations, this paper uses the classical minimum distance (MD) estimator and the principal components instrumental variables (PCIV) estimator. The PCIV estimator is different from conventional IV estimators for structural equations in that nonlinear functions of exogenous and/or predetermined variables are used as instruments. Simulation results comparing the estimator efficiency of the MD and PCIV estimators are reported in this paper. The results indicate that the MD and PCIV estimators are complementary to each other. The estimation methods proposed in this paper are applied to the Japanese export and GDP data to study the effect of export growth rate on that of GDP.
\end{abstract}

\section{INTRODUCTION}

Structural simultaneous equations have been one of the major research themes in econometrics since the 1950 's, ${ }^{1}$ although research on simultaneous equations has attracted relatively less attention these days partly as a result of a perceived failure in large macroeconometric models and partly because of criticisms of simultaneous equations modeling, following work by Lucas (1976) and Sims (1980). However, large-scale simultaneous equations models are cur-

I thank Don Andrews and two referees for very thoughtful comments and suggestions that I believe greatly improved this paper. Part of this paper was written while I was visiting the Department of Economics, University of Southern California. I am grateful to the faculty and staff of the department, especially Quang Vuong, Isabelle Perrigne, Cheng Hsiao, and Young Miller, for their support and hospitality. I also benefited from discussion with participants of econometrics seminars at ASU, UCLA, and UC Riverside. I thank Kangmo Choi for providing research assistance. This paper was financially supported by a research grant from Kookmin University. Some mathematical expressions in this paper were obtained with the help of the software Scientific WorkPlace ${ }^{\mathrm{TM}}$. Address correspondence to: In Choi, Department of Economics, Hong Kong University of Science and Technology, Clear Water Bay, Kowloon, Hong Kong; e-mail: inchoi@ust.hk. 
rently in widespread empirical use for forecasting and policy analyses throughout the world, especially in such nonacademic institutions as private banks and government economics departments, and small-scale simultaneous equations models are still used for academic research. Furthermore, the instrumental variables (IV) estimation methods used for structural equations are quite popular in both empirical and theoretical research. A recent surge of interest in IV estimation with weak instruments (e.g., Bekker, 1994; Bound, Jaeger, and Baker, 1995; Angrist and Krueger, 1995) demonstrates the relevance of the IV estimation methods to empirical analyses.

Prior to estimating the simultaneous equations, it is important to examine the identifiability of the equations. Phillips (1989), Choi and Phillips (1992, 1997), and Staiger and Stock (1997) focus on a single structural equation that is either unidentified or nearly unidentified and show that the conventional estimators for the structural equation do not have standard distributions, even asymptotically. Phillips (1989) and Choi and Phillips (1992, 1997) study the case where the rank condition for identification fails at any sample size, and Staiger and Stock (1997) examine the case where the equation is totally unidentified as sample size goes to infinity. In addition, Wang and Zivot (1998) and Zivot, Startz, and Nelson (1998) study the confidence interval of coefficient estimates for nearly unidentified structural equations by using the methods of Dufour (1997).

Phillips (1989), Choi and Phillips (1992, 1997), and Staiger and Stock (1997) prove how seriously unidentifiability affects the structural equation estimation and, thereby, demonstrate how difficult it is to build a proper structural equation. By contrast, this paper introduces structural equations that do not satisfy the rank and/or order condition(s) for identification but still are identifiable. These equations will be called seemingly unidentified structural equations, because the conventional identification conditions fail for these equations but the equations are still identifiable. The key to the identifiability of these equations is that the right-hand-side endogenous variables undergo structural changes with respect to the exogenous and/or predetermined variables. In the light of prevalent structural changes among economic variables, the structural equations studied here may be of much empirical relevance. Furthermore, the structural equations studied here can be used either for cross-section data or for time series.

Structural changes in a much simpler form than in this paper are also used for the IV estimation in Bekker and van der Ploeg (1999), though they do not make it explicit that their estimation methods utilize structural changes. But the structural changes in their work are simple mean shifts, which enables them to use dummy variables as instruments. Moreover, the model they study is in sharp contrast to ours in that it is identified in the conventional sense and has an infinite number of instruments as sample size grows to infinity. Angrist and Krueger (1995) is also related to this paper in that sample splitting is used for the IV estimation. But these authors' regression model is an identified one in the conventional sense, and the purpose of the sample splitting is to reduce bias, not to detect structural changes as in this paper. 
To estimate the seemingly unidentified structural equations, we use a minimum distance (MD) estimator. This estimator is obtained by using identifying relations for structural coefficients and reduced form parameter estimators. ${ }^{2}$ The MD estimator is simple and intuitively appealing, and its efficiency can be considered within the framework of the MD estimation.

We also introduce a principal components instrumental variables (PCIV) estimator. The PCIV estimator uses principal components of the projected regressor matrix and is basically similar to the principal components OLS estimator except that instruments are used for the PCIV estimator. The PCIV estimator can estimate a linear combination of structural parameters under different data regimes. Therefore, each regime provides a linear combination of structural parameters, and the PCIV estimator for the structural parameters is defined as a solution of these equations. ${ }^{3}$ A major characteristic of the PCIV estimator is that its asymptotic efficiency is not affected by the instruments chosen as long as the instruments are uncorrelated with the error terms of the structural equations.

The PCIV estimator is different from conventional IV estimators for structural equations in that nonlinear functions of exogenous and/or predetermined variables are used as instruments. This practice of using higher order moments of observed data has been prevalent in the literature of regressions with measurement error (cf. Lewbel, 1997; Dagenais and Dagenais, 1995). Using nonlinear functions of instruments in a nonparametric manner is also considered in the literature of nonlinear IV estimation (cf. Newey, 1990).

The MD and PCIV estimators are shown to have the same asymptotic distribution in a special case. But the asymptotic equivalence of the two estimators in general could not be derived in this paper.

Simulation results comparing the efficiency of the MD and PCIV estimators under known structural change points are reported. In addition, how misspecification of structural change points affects the estimators is studied by simulation. Simulation results for the MD and PCIV estimators using estimated structural change points are also presented.

The estimation methods proposed in this paper are applied to the Japanese export and gross domestic product (GDP) data to study the effect of export growth rate on that of GDP. The results reveal that exports had an accelerating multiplier effect on the GDP growth in Japan during the sample period 1960:Q2-1998:Q3.

The plan of the paper is as follows. Section 2 introduces seemingly unidentified structural equations and assumptions required for subsequent analyses. Section 3 studies the MD estimator and its asymptotic distributions. Section 4 introduces the PCIV estimator and derives its asymptotic distributions. Section 5 contains simulation results for estimator efficiency. Section 6 presents an empirical example. Section 7 contains a summary of the paper and some further remarks. All proofs are relegated to the Appendix.

The following notation will be used throughout the paper. For matrix $A, P_{A}=$ $A\left(A^{\prime} A\right)^{-1} A^{\prime}$ and $Q_{A}=I-P_{A}$. 


\section{THE MODELS AND ASSUMPTIONS}

\subsection{A Structural Equation under Rank Condition Failure}

Suppose that a true structural relation is represented by the regression equation

$y_{1 i}=y_{2 i}^{\prime} \beta+z_{1 i}^{\prime} \gamma+u_{i} \quad(i=1, \ldots, N)$,

where $y_{1 i}$ and $y_{2 i}(n \times 1)$ denote $n+1$ endogenous variables, $z_{1 i}\left(k_{1} \times 1\right)$ is a vector of $k_{1}$ exogenous and/or predetermined variables, and $u_{i}$ is a disturbance term.

Letting $z_{2 i}\left(k_{2} \times 1\right)$ be a vector of exogenous and/or predetermined variables excluded from equation (1), write the reduced form equations for the structural equation (1) as

$y_{1 i}=\pi_{1}^{\prime} z_{1 i}+\pi_{2}^{\prime} z_{2 i}+v_{1 i}$

and

$y_{2 i}=\Pi_{1}^{\prime} z_{1 i}+\Pi_{2}^{\prime} z_{2 i}+v_{2 i}$.

Letting $e_{i}^{\prime}=\left[\begin{array}{ll}u_{i} & v_{2 i}^{\prime}\end{array}\right]$, assume

Assumption 1. $E\left(e_{i} e_{k}^{\prime}\right)=I(i=k) \Sigma$, where $I(\cdot)$ is an indicator function, $\Sigma=$ $\left[\begin{array}{ll}\sigma_{u u} & \Sigma_{u v} \\ \Sigma_{v u} & \Sigma_{v v}\end{array}\right]$, and $\Sigma_{u v} \neq 0$.

Under this assumption, $e_{i}$ are uncorrelated, and $y_{2 i}$ and $u_{i}$ are correlated for all $i$.

The identifying relations connecting the parameters of the structural equation (1) and the reduced form equations (2) and (3) are

$\pi_{1}-\Pi_{1} \beta=\gamma$,

$\pi_{2}-\Pi_{2} \beta=0$.

Thus, when the rank condition

$\operatorname{rank}\left(\Pi_{2}\right)=n \quad\left(k_{2} \geq n\right)$,

holds, the structural parameter vector $\left(\beta^{\prime}, \gamma^{\prime}\right)^{\prime}$ is identifiable in the sense that the structural parameters can be recovered from the reduced form parameters, which are almost always estimable.

But it is assumed here that the following polar opposite case of the rank condition (4) holds. ${ }^{4}$

Assumption 2. $\Pi_{2}=0$.

Under this assumption and specification (3), the coefficient vector $\beta$ is totally unidentified. Moreover, the coefficient vector $\gamma$ is also unidentified under this assumption unless $\Pi_{1}=0$. Under Assumption 2, Phillips (1989) and Choi and Phillips (1992) show that the IV estimators using $z_{1 i}$ and $z_{2 i}$ as instruments are 
inconsistent. The main reason for this is that $z_{2 i}$ is not a valid instrument for $y_{2 i}$ under Assumption 2.

However, in addition to Assumption 2, we make the following assumption.

Assumption 3.

$\Pi_{1}=\Pi_{11}$ when $i \in \mathcal{G}_{1}$ and $\Pi_{1}=\Pi_{12}$ when $i \in \mathcal{G}_{2}$,

where $\Pi_{11} \neq \Pi_{12}, \mathcal{G}_{1} \cup \mathcal{G}_{2}=\{1, \ldots, N\}, \mathcal{G}_{1} \cap \mathcal{G}_{2}=\varnothing$, and the numbers of elements of $\mathcal{G}_{1}$ and $\mathcal{G}_{2}$ are $N_{1}$ and $N_{2}$, respectively.

Under Assumptions 2 and 3,

$y_{2 i}=\Pi_{11}^{\prime} z_{1 i}+v_{2 i}, \quad i \in \mathcal{G}_{1}$,

$y_{2 i}=\Pi_{12}^{\prime} z_{1 i}+v_{2 i}, \quad i \in \mathcal{G}_{2}$.

That is, the endogenous variables $y_{2 i}$ undergo a structural change with respect to their relation to the exogenous and/or predetermined variables $z_{1 i}$ under Assumptions 2 and 3 .

The identifying relations connecting the structural equation (1) and the reduced form equations (2) and (5) under Assumption 3 are

$\pi_{11}-\Pi_{11} \beta=\gamma$

$\pi_{12}-\Pi_{12} \beta=\gamma$

where $\pi_{1 j}$ denotes the coefficient $\pi_{1}$ under $i \in \mathcal{G}_{j}$. These equations show that the structural parameters are identifiable even under the rank condition failure, because equations (6) and (7) can be solved with respect to the parameter vectors $\beta$ and $\gamma$ when the following assumption is satisfied.

Assumption 4. $\operatorname{rank}\left(\begin{array}{cc}I_{k_{1}} & \Pi_{11} \\ I_{k_{1}} & \Pi_{12}\end{array}\right)=n+k_{1}$.

Note that Assumption 4 implicitly requires $n \leq k_{1}$.

The key element in identifying the structural equation (1) is that the reduced form equation for the endogenous variable $y_{2 i}$ undergoes a structural change, whereas the structural equation does not. Structural equations are usually based on economic theory or reasoning that is assumed to hold true for any economic agent and in any time period. Economic theory seldom predicts structural changes. Thus, assuming parameter constancy for the structural equation may not be too unreasonable unless significant and widespread social changes (e.g., changes in economic system, revolutions, etc.) are known to occur. However, reduced form equations merely signify data generating processes for which structural changes are often observed. A well known example is Perron (1989). Notwithstanding these arguments, testing whether the identifiability conditionsabsence of structural changes for the structural equation and the presence of a structural change in the reduced form equation-hold for a given data set is important in empirical practices and, therefore, needs to be pursued. 
We have considered the structural equation under the rank condition failure, but this is also related to a general IV estimation problem. Write equations (1) and (3) under Assumption 2 as

$y_{1 i}=w_{i}^{\prime} \delta+u_{i}$,

where $w_{i}=\left[y_{2 i}^{\prime}, z_{1 i}^{\prime}\right]^{\prime}, \delta=\left[\beta^{\prime}, \gamma^{\prime}\right]^{\prime}$, and

$\left(\begin{array}{c}y_{2 i} \\ z_{1 i}\end{array}\right)=\left(\begin{array}{c}\Pi_{1}^{\prime} \\ I_{k_{1}}\end{array}\right) z_{1 i}+\left(\begin{array}{c}v_{2 i} \\ 0\end{array}\right)$

or equivalently

$w_{i}=\Xi^{\prime} z_{1 i}+v_{i}$.

Written in this way, equations (1) and (3) under Assumption 2 denote the IV regression with insufficient number of instruments. It is inferred from the discussions up to now that the problem of insufficient number of instruments in the IV estimation can be overcome where there is a structural change in the relation between the endogenous variables and instruments. For this reason, estimation theory that will be developed in subsequent sections can equally be applied to IV estimation with insufficient number of instruments.

A time series example of a seemingly unidentified structural equation under the rank condition failure is

$y_{1 i}=\beta y_{2 i}+\gamma y_{2(i-1)}+u_{i}$

with

$y_{2 i}=\Pi_{11} y_{2(i-1)}+v_{2 i}, \quad\left|\Pi_{11}\right|<1, \quad i \in \mathcal{G}_{1}$,

$y_{2 i}=\Pi_{12} y_{2(i-1)}+v_{2 i}, \quad\left|\Pi_{12}\right|<1, \quad i \in \mathcal{G}_{2}$,

and

$\operatorname{rank}\left(\begin{array}{cc}1 & \Pi_{11} \\ 1 & \Pi_{12}\end{array}\right)=2$

Equations (8) and (9) signify a typical distributed lag model where the righthand-side variable of the structural equation follows an $\operatorname{AR}(1)$ process. One may usually be interested in the value of $\beta+\gamma$, which is called the long-run multiplier. When there is no structural change in the AR(1) process, the structural equation (8) is unidentifiable. But a structural change as in equation (9) makes the structural equation identifiable. 


\subsection{A Structural Equation under Order Condition Failure}

Consider a structural equation

$y_{1 i}=y_{2 i}^{\prime} \beta+z_{1 i}^{\prime} \gamma+z_{2 i}^{\prime} \rho+u_{i}$,

where $y_{2 i}, z_{1 i}$, and $u_{i}$ are as defined in Section 2.1 and $z_{2 i}\left(k_{2} \times 1\right)$ is a vector of exogenous and/or predetermined variables. We treat this equation separately from equation (1) because the regressor $z_{2 i}$ is included in the regression equation (10) regardless of the true value of the parameter vector $\rho$.

The identifying relations connecting the parameters of the structural equation (10) and the reduced form equations (2) and (3) are

$\pi_{1}-\Pi_{1} \beta=\gamma$,

$\pi_{2}-\Pi_{2} \beta=\rho$.

The condition $\rho=0$ is a necessary condition for the identification of equation (10) and has been called the order condition for identification. ${ }^{5}$ When this condition is violated, it is obvious that the IV estimator using $z_{1 i}$ and $z_{2 i}$ as instruments is inconsistent. However, it is assumed in this paper that there are no restrictions on $\rho$ as in the following assumption.

Assumption 5. $\rho \in R^{k_{2}}$.

To make the structural parameters identified, however, we assume that Assumption 3 and the following assumption hold.

Assumption 6. $\operatorname{rank}\left(\begin{array}{ccc}I_{k_{1}} & 0 & \Pi_{11} \\ I_{k_{1}} & 0 & \Pi_{12} \\ 0 & I_{k_{2}} & \Pi_{2}\end{array}\right)=n+k_{1}+k_{2}$.

Under this assumption and Assumption 3, equations (6), (7), and (11) can be solved with respect to the structural parameters $\beta, \gamma$, and $\rho$. For simplicity, it is assumed here that the reduced form parameter $\Pi_{2}$ does not undergo structural changes. Extending the results of this paper to the case where $\Pi_{2}$ changes over regimes $\mathcal{G}_{1}$ and $\mathcal{G}_{2}$ is straightforward. In addition, Assumption 6 implicitly requires $n \leq k_{1}$.

Assumption 6 replaces Assumption 4, so that it is not required here anymore. Furthermore, $\Pi_{2}$ may take any value as long as it satisfies Assumption 6, and hence Assumption 2 is not needed in this section. In summary, this section requires Assumptions 1, 3, 5, and 6.

Equation (10) with the reduced form equation (3) may be interpreted as an IV regression model with insufficient number of instruments, as in Section 2.1. Thus, the IV regression model can be identified when the endogenous variables in the model undergo a structural change with respect to a set of instru- 
ments, the number of which is greater than or equal to the number of the endogenous variables.

As a time series example of a seemingly unidentified structural equation under the order condition failure, consider

$y_{1 i}=\beta y_{2 i}+\gamma y_{2(i-1)}+\rho x_{1 i}+u_{i}$,

where $x_{1 i}$ is a strictly exogenous variable,

$y_{2 i}=\Pi_{11} y_{2(i-1)}+\Pi_{2} x_{1 i}+v_{2 i}, \quad\left|\Pi_{11}\right|<1, \quad i \in \mathcal{G}_{1}$,

$y_{2 i}=\Pi_{12} y_{2(i-1)}+\Pi_{2} x_{1 i}+v_{2 i}, \quad\left|\Pi_{12}\right|<1, \quad i \in \mathcal{G}_{2}$

and

$\operatorname{rank}\left(\begin{array}{ccc}1 & 0 & \Pi_{11} \\ 1 & 0 & \Pi_{12} \\ 0 & 1 & \Pi_{2}\end{array}\right)=3$.

Equation (12) is a typical structural equation that violates the order condition. However, with a structural change as in equation (13), the structural equation (12) would be identified. Parameter $\rho$ may take any value in this example.

\section{MINIMUM DISTANCE ESTIMATION}

\subsection{Minimum Distance Estimator}

This section considers MD estimators for the structural parameters in equations (1) and (10). The objective function for the MD estimation of the parameter vector $\delta=\left(\beta^{\prime}, \gamma^{\prime}\right)^{\prime}$ is a weighted product of the sample version of the identifying relations (6) and (7). That is, the MD estimator of the coefficient vector $\delta=\left(\beta^{\prime}, \gamma^{\prime}\right)^{\prime}$ in the regression equation (1) is obtained by minimizing the objective function

$R(\delta)=(\hat{\pi}-\hat{\Pi} \delta)^{\prime} \Upsilon_{\delta}^{-1}(\hat{\pi}-\hat{\Pi} \delta)$

with respect to the parameter vector $\delta$, where $Y_{\delta}^{-1}$ is a weight matrix. Here, letting $Z_{1 j}=\left[z_{1 i}^{\prime}\right]_{i \in \mathcal{G}_{j}}, Y_{1 j}=\left[y_{1 i}\right]_{i \in \mathcal{G}_{j}}$, and $Y_{2 j}=\left[y_{2 i}^{\prime}\right]_{i \in \mathcal{G}_{j}}(j=1,2)$,

$$
\begin{aligned}
\hat{\pi} & =\left[\begin{array}{c}
\hat{\pi}_{11} \\
\hat{\pi}_{12}
\end{array}\right], \quad \hat{\Pi}=\left[\begin{array}{ll}
\hat{\Pi}_{11} & I_{k_{1}} \\
\hat{\Pi}_{12} & I_{k_{1}}
\end{array}\right], \\
\hat{\pi}_{1 j} & =\left(Z_{1 j}^{\prime} Z_{1 j}\right)^{-1} Z_{1 j}^{\prime} Y_{1 j}, \hat{\Pi}_{1 j}=\left(Z_{1 j}^{\prime} Z_{1 j}\right)^{-1} Z_{1 j}^{\prime} Y_{2 j} .
\end{aligned}
$$


The best efficiency of the MD estimator is attained when ${ }^{6}$ $\Upsilon_{\delta}=\frac{1}{\sigma_{u u}} E(\hat{\pi}-\hat{\Pi} \delta)(\hat{\pi}-\hat{\Pi} \delta)^{\prime}=\left[\begin{array}{cc}\left(Z_{11}^{\prime} Z_{11}\right)^{-1} & 0 \\ 0 & \left(Z_{12}^{\prime} Z_{12}\right)^{-1}\end{array}\right]$.

Note that the second equality in equation (14) follows from the relation $\hat{\pi}-\hat{\Pi} \delta=A U$, where

$A=\left[\begin{array}{cc}\left(Z_{11}^{\prime} Z_{11}\right)^{-1} Z_{11}^{\prime} & 0 \\ 0 & \left(Z_{12}^{\prime} Z_{12}\right)^{-1} Z_{12}^{\prime}\end{array}\right]$

and $U=\left[u_{1}, \ldots, u_{N}\right]^{\prime}$. The resulting MD estimator of $\delta$ is written as

$\hat{\delta}_{\mathrm{MD}}=\left(\hat{\Pi}^{\prime} \Upsilon_{\delta}^{-1} \hat{\Pi}\right)^{-1} \hat{\Pi}^{\prime} \Upsilon_{\delta}^{-1} \hat{\pi}$.

Analogously, letting $Z_{2 j}=\left[z_{2 i}^{\prime}\right]_{i \in \mathcal{G}_{j}}, Z_{1}=\left[z_{1 i}^{\prime}\right]_{i=1, \ldots, N}, Z_{2}=\left[z_{2 i}^{\prime}\right]_{i=1, \ldots, N}$, $Y_{1}=\left[y_{1 i}\right]_{i=1, \ldots, N}$, and $Y_{2}=\left[y_{2 i}^{\prime}\right]_{i=1, \ldots, N}$, the MD estimator of the parameter vector $\phi=\left(\beta^{\prime}, \gamma^{\prime}, \rho^{\prime}\right)^{\prime}$ for the regression equation (10) is defined as

$\bar{\phi}_{\mathrm{MD}}=\left(\bar{\Pi}^{\prime} \Upsilon_{\phi}^{-1} \bar{\Pi}\right)^{-1} \bar{\Pi}^{\prime} \Upsilon_{\phi}^{-1} \bar{\pi}$,

where

$$
\begin{aligned}
\bar{\pi} & =\left[\begin{array}{c}
\bar{\pi}_{11} \\
\bar{\pi}_{12} \\
\bar{\pi}_{2}
\end{array}\right], \quad \bar{\Pi}=\left[\begin{array}{ccc}
\bar{\Pi}_{11} & I_{k_{1}} & 0 \\
\bar{\Pi}_{12} & I_{k_{1}} & 0 \\
\bar{\Pi}_{2} & 0 & I_{k_{2}}
\end{array}\right], \quad \Upsilon_{\phi}=\left[\begin{array}{ccc}
\Upsilon_{\phi 11} & \Upsilon_{\phi 12} & \Upsilon_{\phi 13} \\
\Upsilon_{\phi 12}^{\prime} & \Upsilon_{\phi 22} & \Upsilon_{\phi 23} \\
\Upsilon_{\phi 13}^{\prime} & \Upsilon_{\phi 23}^{\prime} & \Upsilon_{\phi 33}
\end{array}\right], \\
\bar{\pi}_{1 j} & =\left(Z_{1 j}^{\prime} Q_{Z_{2 j}} Z_{1 j}\right)^{-1} Z_{1 j}^{\prime} Q_{Z_{2 j}} Y_{1 j} \quad(j=1,2), \\
\bar{\pi}_{2} & =\left(Z_{2}^{\prime} Q_{Z_{1}} Z_{2}\right)^{-1} Z_{2}^{\prime} Q_{Z_{1}} Y_{1}, \\
\bar{\Pi}_{1 j} & =\left(Z_{1 j}^{\prime} Q_{Z_{2 j}} Z_{1 j}\right)^{-1} Z_{1 j}^{\prime} Q_{Z_{2 j}} Y_{2 j} \quad(j=1,2), \\
\bar{\Pi}_{2} & =\left(Z_{2}^{\prime} Q_{Z_{1}} Z_{2}\right)^{-1} Z_{2}^{\prime} Q_{Z_{1}} Y_{2}, \\
\Upsilon_{\phi 11} & =\left(Z_{11}^{\prime} Q_{Z_{21}} Z_{11}\right)^{-1}, \quad \Upsilon_{\phi 22}=\left(Z_{12}^{\prime} Q_{Z_{22}} Z_{12}\right)^{-1}, \quad \Upsilon_{\phi 33}=\left(Z_{2}^{\prime} Q_{Z_{1}} Z_{2}\right)^{-1}, \\
\Upsilon_{\phi 12} & =0, \quad \Upsilon_{\phi 13}=\Upsilon_{\phi 23}=-\left(Z_{1}^{\prime} Z_{1}\right)^{-1} Z_{1}^{\prime} Z_{2}\left(Z_{2}^{\prime} Q_{Z_{1}} Z_{2}\right)^{-1} .
\end{aligned}
$$

It follows from the relation $\bar{\pi}=\bar{\Pi} \phi+B U$, where

$$
B=\left[\begin{array}{c}
\left(Z_{11}^{\prime} Q_{Z_{21}} Z_{11}\right)^{-1} Z_{11}^{\prime} Q_{Z_{21}}\left[I_{N_{1}}, 0\right] \\
\left(Z_{12}^{\prime} Q_{Z_{22}} Z_{12}\right)^{-1} Z_{12}^{\prime} Q_{Z_{22}}\left[0, I_{N_{2}}\right] \\
\left(Z_{2}^{\prime} Q_{Z_{1}} Z_{2}\right)^{-1} Z_{2}^{\prime} Q_{Z_{1}}
\end{array}\right],
$$

that $\Upsilon_{\phi}=B B^{\prime}$. 


\subsection{Asymptotic Distribution Theory}

This section reports asymptotic distributions of the MD estimators introduced in the last section. For the asymptotic distributions of the MD estimators defined in (16) and (17), the following assumption is required.

Assumption 7. As $N, N_{j} \rightarrow \infty(j=1,2)$,

(a) $\left(1 / N_{j}\right) Z_{1 j}^{\prime} Z_{1 j} \stackrel{p}{\rightarrow} M_{z_{1 j} z_{1 j}}$, where $M_{z_{1 j} z_{1 j}}$ is a positive definite nonrandom matrix;

(b)

$$
\frac{1}{N_{j}} Z^{j \prime} Z^{j} \stackrel{p}{\rightarrow}\left[\begin{array}{ll}
M_{z_{1 j} z_{1 j}} & M_{z_{1 j} z_{2 j} j} \\
M_{z_{2 j} z_{1 j}} & M_{z_{2 j} z_{2 j}}
\end{array}\right] \begin{gathered}
k_{1} \\
k_{2}
\end{gathered},
$$

where $Z^{j}=\left[Z_{1 j}, Z_{2 j}\right]$ and the probability limit of $\left(1 / N_{j}\right) Z^{j} Z^{j}$ is a positive definite nonrandom matrix and partitioned conformably;

(c) $\left(1 / N_{j}\right) Z^{j \prime} V_{1 j} \stackrel{p}{\rightarrow} 0$ and $\left(1 / N_{j}\right) Z^{j \prime} V_{2 j} \stackrel{p}{\rightarrow} 0$, where $V_{1 j}=\left[v_{1 i}\right]_{i \in \mathcal{G}_{j}}$ and $V_{2 j}=$ $\left[v_{2 i}^{\prime}\right]_{i \in \mathcal{G}_{j}}$

(d) $\left(N_{j} / N\right) \rightarrow d_{j}$ where $0<d_{j}<1$;

(e) For $K=A$ or $B$,

$$
\sqrt{N} K U \stackrel{d}{\rightarrow} N\left(0, \sigma_{u u} \operatorname{plim}_{N \rightarrow \infty} N K K^{\prime}\right),
$$

where the matrices $A$ and $B$ are given in equations (15) and (18), respectively.

Assumption 7 follows from the law of large numbers and the central limit theorem when specific conditions are given to the involved random variables.

The asymptotic distributions of the minimum distance estimators $\hat{\delta}_{\mathrm{MD}}$ and $\bar{\phi}_{\mathrm{MD}}$ are reported in the following theorem.

THEOREM 1. (a) Under Assumptions $1-4$ and 7 , as $N, N_{j} \rightarrow \infty(j=1,2)$, $\sqrt{N}\left(\hat{\delta}_{\mathrm{MD}}-\delta\right) \stackrel{d}{\rightarrow} N\left(0, \sigma_{u u} \Omega^{-1}\right)$, where $\Omega=\left[\begin{array}{ll}\Pi_{11} & I_{k_{1}} \\ \Pi_{12} & I_{k_{1}}\end{array}\right]^{\prime}\left[\begin{array}{cc}\frac{1}{d_{1}} M_{z_{11} z_{11}}^{-1} & 0 \\ 0 & \frac{1}{d_{2}} M_{z_{12} z_{12}}^{-1}\end{array}\right]^{-1}\left[\begin{array}{cc}\Pi_{11} & I_{k_{1}} \\ \Pi_{12} & I_{k_{1}}\end{array}\right]$.

(b) Assume that the matrix $D$ defined subsequently is nonsingular. Under Assumptions 1, 3, and 5-7, as $N, N_{j} \rightarrow \infty(j=1,2)$,

$\sqrt{N}\left(\bar{\phi}_{\mathrm{MD}}-\delta\right) \stackrel{d}{\rightarrow} N\left(0, \sigma_{u u} \bar{\Omega}^{-1}\right)$, 
where

$$
\begin{aligned}
& \bar{\Omega}=\left[\begin{array}{ccc}
\Pi_{11} & I_{k_{1}} & 0 \\
\Pi_{12} & I_{k_{1}} & 0 \\
\Pi_{2} & 0 & I_{k_{2}}
\end{array}\right]^{\prime} D^{-1}\left[\begin{array}{ccc}
\Pi_{11} & I_{k_{1}} & 0 \\
\Pi_{12} & I_{k_{1}} & 0 \\
\Pi_{2} & 0 & I_{k_{2}}
\end{array}\right], \\
& D=\left[\begin{array}{ccc}
\frac{1}{d_{1}} M_{z_{11} z_{21}}^{-1} & 0 & -M_{z_{1} z_{1}}^{-1} M_{z_{1} z_{2}} M_{z_{2} z_{1}}^{-1} \\
0 & \frac{1}{d_{2}} M_{z_{12} z_{22}}^{-1} & -M_{z_{1} z_{1}}^{-1} M_{z_{1} z_{2}} M_{z_{2} z_{1}}^{-1} \\
-M_{z_{2} \bullet z_{1}}^{-1} M_{z_{2} z_{1}} M_{z_{1} z_{1}}^{-1} & -M_{z_{2} \bullet z_{1}}^{-1} M_{z_{2} z_{1}} M_{z_{1} z_{1}}^{-1} & M_{z_{2} z_{1}}^{-1}
\end{array}\right] \text {, } \\
& M_{a \bullet b}=M_{a a}-M_{a b} M_{b b}^{-1} M_{b a}, \\
& \text { and }\left[\begin{array}{ll}
M_{z_{1} z_{1}} & M_{z_{1} z_{2}} \\
M_{z_{2} z_{1}} & M_{z_{2} z_{2}}
\end{array}\right] \text { is the probability limit of }(1 / N) Z^{\prime} Z \text { with } Z=\left[Z_{1}, Z_{2}\right] \text {. }
\end{aligned}
$$

\section{Remarks}

(a) This theorem shows that the MD estimators are consistent and normally distributed in the limit.

(b) When Assumptions 4 and 6 do not hold, the variance-covariance matrices in Theorem 1 become singular, which indicates the lack of identifiability.

(c) In the special case $n=k_{1}=1$, the variance-covariance matrix $\Omega$ becomes

$$
\sigma_{u u}\left[\begin{array}{cc}
\frac{d_{1} M_{z_{11} z_{11}}+d_{2} M_{z_{12} z_{12}}}{d_{1} M_{z_{11} z_{11}} d_{2} M_{z_{12} z_{12}}\left(\Pi_{12}-\Pi_{11}\right)^{2}} & -\frac{\Pi_{11} d_{1} M_{z_{11} z_{11}}+\Pi_{12} d_{2} M_{z_{12} z_{12}}}{d_{1} M_{z_{11} z_{11}} d_{2} M_{z_{12} z_{12}}\left(\Pi_{12}-\Pi_{11}\right)^{2}} \\
-\frac{\Pi_{11} d_{1} M_{z_{11} z_{11}}+\Pi_{12} d_{2} M_{z_{12} z_{12}}}{d_{1} M_{z_{11} z_{11}} d_{2} M_{z_{12} z_{12}}\left(\Pi_{12}-\Pi_{11}\right)^{2}} & \frac{\Pi_{11}^{2} d_{1} M_{z_{11} z_{11}}+\Pi_{12}^{2} d_{2} M_{z_{12} z_{12}}}{d_{1} M_{z_{11} z_{11}} d_{2} M_{z_{12} z_{12}}\left(\Pi_{12}-\Pi_{11}\right)^{2}}
\end{array}\right] .
$$

Several observations emerge from the asymptotic variance-covariance matrix (19). First, the asymptotic variances of $\hat{\beta}_{\mathrm{MD}}$ and $\hat{\gamma}_{\mathrm{MD}}$ decrease as $\left(\Pi_{12}-\Pi_{11}\right)^{2}$ increases. That is, more abrupt structural changes yield higher asymptotic efficiency. Second, the variance and covariance formulae for $\hat{\beta}_{\mathrm{MD}}$ and $\hat{\gamma}_{\mathrm{MD}}$ break down when $\Pi_{11}=\Pi_{12}$, which indicates the lack of identifiability.

\section{PRINCIPAL COMPONENTS IV ESTIMATION}

\subsection{Basic Ideas and Assumptions}

The coefficients for the structural equation under regime $j(j=1,2)$,

$y_{1 i}=y_{2 i}^{\prime} \beta+z_{1 i}^{\prime} \gamma+u_{i}, \quad i \in \mathcal{G}_{j}$ 
with

$y_{2 i}=\Pi_{1 j}^{\prime} z_{1 i}+v_{2 i}, \quad i \in \mathcal{G}_{j}$,

cannot be estimated by the two-stage least squares estimation as discussed in Phillips (1989) and Choi and Phillips (1992). The two-stage least squares estimation fails mainly because $\hat{y}_{2 i}\left(=\hat{\Pi}_{1 j}^{\prime} z_{1 i} ; \hat{\Pi}_{1 j}\right.$ is the ordinary least squares [OLS] estimator) and $z_{1 i}$ are collinear. But when there exists multicollinearity among regressors, it is well known ${ }^{7}$ that the principal component regression can still estimate a linear combination of the coefficient vector. This implies that we can estimate two linear combinations of the structural coefficient vector from the two data regimes $\mathcal{G}_{1}$ and $\mathcal{G}_{2}$ and use them to estimate the structural coefficient vector.

In using the principal component regression for the regression equation (20), there are two problems that deserve our attention. First, the regressor $y_{2 i}$ and the disturbance term $u_{i}$ are correlated. Second, $z_{2 i}$ may not be available for use as instruments because of Assumption 2. To resolve these problems, we will use the principal component regression using instrument variables, which we call principal component instrumental variables (PCIV) regression. As instruments, nonlinear functions of $z_{1 i}, H\left(z_{1 i}\right)=h_{i},{ }^{8}$ are used because other instruments may not be available in practice. ${ }^{9}$ It is assumed that the number of elements of the vector $h_{i}$ is greater than or equal to $n$ (the number of elements in vector $y_{2 i}$ ) for the condition of sufficient number of instruments to be satisfied. In the literature of regressions with measurement error, higher order moments of observed data have long been used as instruments. ${ }^{10}$ In this paper, this practice is adopted to estimate structural equations.

In the same manner, the PCIV regression method can be used to estimate the structural coefficient vector for equation (10). In this case, we use $F\left(z_{1 i}, z_{2 i}\right)=$ $f_{i}$ as instruments for $y_{2 i}$, where $F(\cdot, \cdot)$ is a nonlinear function and the number of elements of $f_{i}$ is greater than or equal to that of $y_{2 i}$.

Now we introduce assumptions required for the development of distribution theory. The instrument matrices that we will use for equations (1) and (10) are $G=\left[\begin{array}{c}G_{1} \\ G_{2}\end{array}\right], \quad G_{j}=\left[h_{i}^{\prime}, z_{1 i}^{\prime}\right]_{i \in \mathcal{G}_{j}} \quad$ and $\quad \bar{G}=\left[\begin{array}{c}\bar{G}_{1} \\ \bar{G}_{2}\end{array}\right]$, $\bar{G}_{j}=\left[f_{i}^{\prime}, z_{1 i}^{\prime}, z_{2 i}^{\prime}\right]_{i \in \mathcal{G}_{j}} \quad(j=1,2)$,

respectively, for which the following assumption is made for the PCIV estimation.

Assumption 8. As $N, N_{j} \rightarrow \infty(j=1,2)$,

(a) $\left(1 / N_{j}\right) G_{j}^{\prime} G_{j} \stackrel{p}{\rightarrow} M_{G_{j} G_{j}}=\left[\begin{array}{cc}M_{h_{j} h_{j}} & M_{h_{j} z_{1 j}} \\ M_{z_{1 j} h_{j}} & M_{z_{1 j} z_{1 j}}\end{array}\right]$, where $M_{G_{j} G_{j}}$ is a positive definite nonrandom matrix and partitioned conformably; 
(b) $\left(1 / N_{j}\right) \bar{G}_{j}^{\prime} \bar{G}_{j} \stackrel{p}{\rightarrow} M_{\bar{G}_{j} \bar{G}_{j}}=\left[\begin{array}{ccc}M_{f_{j} f_{j}} & M_{f_{j} z_{1 j}} & M_{f_{j} z_{2 j}} \\ M_{z_{1 j} f_{j}} & M_{z_{1 j} z_{1 j}} & M_{z_{1 j} z_{2 j}} \\ M_{z_{2 j} f_{j}} & M_{z_{2 j} z_{1 j}} & M_{z_{2 j} z_{2 j}}\end{array}\right]$, where $M_{\bar{G}_{j} \bar{G}_{j}}$ is a positive definite nonrandom matrix and partitioned conformably;

(c) $\left(1 / N_{j}\right) V_{2 j}^{\prime} L \stackrel{p}{\rightarrow} 0$, where $L=G_{j}$ or $\bar{G}_{j}$;

(d) $\left(N_{j} / N\right) \rightarrow d_{j}$, where $0<d_{j}<\infty$;

(e) for $L=G_{j}$ or $\bar{G}_{j}$,

$$
\frac{1}{\sqrt{N_{j}}} L^{\prime} U \stackrel{d}{\rightarrow} N\left(0, \sigma_{u u} \operatorname{plim}_{N_{j} \rightarrow \infty} \frac{1}{N_{j}} L^{\prime} L\right) .
$$

Parts (a) and (b) of Assumption 8 essentially imply that the law of large numbers holds for the products of the instrument matrices and that their probability limits are positive definite. Note that the instrument matrices are allowed to be stochastic for our asymptotic analysis. Part (c) follows when more specific conditions are given on matrix $V_{2 j}$. For example, $v_{2 i}$ may be either vector martingale difference sequence or vector independent and identically distributed (i.i.d.) sequence. Part (e) follows from the central limit theorem with more specific conditions on the errors and instruments.

\subsection{Principal Components IV Estimator}

This section formally introduces the PCIV estimator for the seemingly unidentified structural equations in Section 2. The principal components OLS regression requires knowing how many nonzero eigenvalues are present in the product of a regressor matrix. Likewise, the PCIV estimator applied to each regime of models (1) and (10) will require the number of nonzero eigenvalues for the product of a projected regressor matrix. The following lemma will be used to find such numbers.

\section{LEMMA 1.}

(a) Under Assumptions 2 and $8, \operatorname{rank}\left(M_{W_{j} G_{j}} M_{G_{j} G_{j}}^{-1} M_{G_{j} W_{j}}\right)=k_{1}$, where $M_{W_{j} G_{j}} M_{G_{j} G_{j}}^{-1} M_{G_{j} W_{j}}=\operatorname{plim}_{N_{j} \rightarrow \infty}\left(1 / N_{j}\right) W_{j}^{\prime} P_{G_{j}} W_{j}$ and $W_{j}=\left[y_{2 i}^{\prime}, z_{1 i}^{\prime}\right]_{i \in \mathcal{G}_{j}}(j=1,2)$.

(b) Under Assumption 8, $\operatorname{rank}\left(M_{\bar{W}_{j}} \bar{G}_{j} M_{\bar{G}_{j}} \bar{G}_{j} M_{\bar{G}_{j} \bar{W}_{j}}\right)=k_{1}+k_{2}$, where $M_{\bar{W}_{j} \bar{G}_{j}} M_{\bar{G}_{j}}^{-1} \bar{G}_{j} M_{\bar{G}_{j} \bar{W}_{j}}=\operatorname{plim}_{N_{j} \rightarrow \infty}\left(1 / N_{j}\right) \bar{W}_{j}^{\prime} P_{\bar{G}_{j}} \bar{W}_{j}$ and $\bar{W}_{j}=\left[y_{2 i}^{\prime}, z_{1 i}^{\prime}, z_{2 i}^{\prime}\right]_{i \in \mathcal{G}_{j}}$ $(j=1,2)$.

To introduce the PCIV estimator, write equation (20) in matrix notation as

$$
\begin{aligned}
Y_{1 j} & =W_{j} \delta+U_{j}, \quad\left(U_{j}=\left[u_{i}\right]_{i \in \mathcal{G}_{j}}\right) \\
& =W_{j} S_{j} S_{j}^{\prime} \delta+U_{j} \\
& =W_{j} S_{j 1} \theta_{j 1}+W_{j} S_{j 2} \theta_{j 2}+U_{j} \\
& =X_{1 j} \theta_{j 1}+X_{2 j} \theta_{j 2}+U_{j}
\end{aligned}
$$


where $S_{j}=\left[\begin{array}{cc}k_{1} & n \\ S_{j 1} & S_{j 2}\end{array}\right]$ is a matrix whose columns are orthonormal eigenvectors of $W_{j}^{\prime} P_{G_{j}} W_{j}, \theta_{j m}=S_{j m}^{\prime} \delta(m=1,2)$ and $X_{m j}=W_{j} S_{j m}$. Here, the eigenvectors are ordered such that

$S_{j}^{\prime} W_{j}^{\prime} P_{G_{j}} W_{j} S_{j}=\operatorname{diag}\left[\lambda_{j 1}, \ldots, \lambda_{j\left(n+k_{1}\right)}\right] \quad$ and $\quad \lambda_{j 1} \geq \cdots \geq \lambda_{j\left(n+k_{1}\right)}$.

Thus, vectors in the matrix $S_{j 1}$ correspond to the $k$ largest eigenvalues $\lambda_{j 1}, \ldots, \lambda_{j k_{1}}$.

Using part (a) of Lemma 1 , the PCIV estimators of $\theta_{j 1}$ and $\theta_{j 2}$ using $G_{j}$ as an instrument matrix are defined as

$\hat{\theta}_{j 1 \mathrm{IV}}=\left(X_{1 j}^{\prime} P_{G_{j}} X_{1 j}\right)^{-1} X_{1 j}^{\prime} P_{G_{j}} Y_{1 j}$

and $\hat{\theta}_{j 2 \mathrm{IV}}=0$, respectively. These are obtained by multiplying the matrix $P_{G_{j}}$ to both sides of equation (21) and running the principal components OLS regression.

Because $X_{1 j}^{\prime} P_{G_{j}} X_{2 j}=0$ because of the orthogonality of eigenvectors, equation (22) can be rewritten as

$\hat{\theta}_{j 1 \mathrm{IV}}=S_{j 1}^{\prime} \delta+\left(X_{1 j}^{\prime} P_{G_{j}} X_{1 j}\right)^{-1} X_{1 j}^{\prime} P_{G_{j}} U_{j}$

But the second term on the right-hand side of equation (23) is asymptotically negligible, and hence we have

$\left[\begin{array}{l}\hat{\theta}_{11 \mathrm{IV}} \\ \hat{\theta}_{21 \mathrm{IV}}\end{array}\right] \cong\left[\begin{array}{l}S_{11}^{\prime} \\ S_{21}^{\prime}\end{array}\right] \delta=\Gamma \delta$.

Applying the principle of minimum distance estimation as in Section 3 gives the PCIV estimator of the vector $\delta$ as ${ }^{11}$

$\hat{\delta}_{\mathrm{PCIV}}=\left(\Gamma^{\prime} \Theta_{\delta}^{-1} \Gamma\right)^{-1} \Gamma^{\prime} \Theta_{\delta}^{-1}\left[\begin{array}{c}\hat{\theta}_{11 \mathrm{IV}} \\ \hat{\theta}_{21 \mathrm{IV}}\end{array}\right]$,

where $\Theta_{\delta}=\left[\begin{array}{cc}\left(X_{11}^{\prime} P_{G_{1}} X_{11}\right)^{-1} & 0 \\ 0 & \left(X_{12}^{\prime} P_{G_{2}} X_{12}\right)^{-1}\end{array}\right]$.

The PCIV estimator for the regression equation (10) is defined in the same way as for the regression equation (1). Write model (10) under $i \in \mathcal{G}_{j}$ as

$$
\begin{aligned}
Y_{1 j} & =\bar{W}_{j} \phi+U_{j} \\
& =\bar{W}_{j} \bar{S}_{j} \bar{S}_{j}^{\prime} \phi+U_{j} \\
& =\bar{W}_{j} \bar{S}_{j 1} \tau_{j 1}+\bar{W}_{j} \bar{S}_{j 2} \tau_{j 2}+U_{j} \\
& =\bar{X}_{1 j} \tau_{j 1}+\bar{X}_{2 j} \tau_{j 2}+U_{j},
\end{aligned}
$$


where $\bar{S}_{j}=\left[\begin{array}{cc}k_{1}+k_{2} & n \\ \bar{S}_{j 1} & \bar{S}_{j 2}\end{array}\right]$ is a matrix whose columns are orthonormal eigenvectors of $\bar{W}_{j}^{\prime} P_{\bar{G}_{j}} \bar{W}_{j}, \bar{S}_{j 1}$ corresponds to the $k_{1}+k_{2}$ largest eigenvalues of $\bar{W}_{j}^{\prime} P_{\bar{G}_{j}} \bar{W}_{j}$, $\tau_{j m}=\bar{S}_{j m}^{\prime} \phi(m=1,2)$ and $\bar{X}_{1 j}=\bar{W}_{j} \bar{S}_{j m}$.

By using part (b) of Lemma 1, the PCIV estimator of $\tau_{j 1}$ is defined as

$\bar{\tau}_{j 1 \mathrm{IV}}=\left(\bar{X}_{1 j}^{\prime} P_{\bar{G}_{j}} \bar{X}_{1 j}\right)^{-1} \bar{X}_{1 j}^{\prime} P_{\bar{G}_{j}} Y_{1 j}$

But because

$\bar{\tau}_{j 1 \mathrm{IV}}=\bar{S}_{j 1}^{\prime} \phi+\left(\bar{X}_{1 j}^{\prime} P_{\bar{G}_{j}} \bar{X}_{1 j}\right)^{-1} \bar{X}_{1 j}^{\prime} P_{\bar{G}_{j}} U_{j}$

and the second term on the right-hand side of equation (25) is negligible, the PCIV estimator of $\phi$ is defined as

$\bar{\phi}_{\mathrm{PCIV}}=\left(\bar{\Gamma}^{\prime} \Theta_{\phi}^{-1} \bar{\Gamma}\right)^{-1} \bar{\Gamma}^{\prime} \Theta_{\phi}^{-1}\left[\begin{array}{c}\bar{\tau}_{11 \mathrm{IV}} \\ \bar{\tau}_{21 \mathrm{IV}}\end{array}\right]$,

where $\bar{\Gamma}=\left[\begin{array}{c}\bar{S}_{11}^{\prime} \\ \bar{S}_{21}^{\prime}\end{array}\right]$ and $\Theta_{\phi}=\left[\begin{array}{cc}\left(\bar{X}_{11}^{\prime} P_{\bar{G}_{1}} \bar{X}_{11}\right)^{-1} & 0 \\ 0 & \left(\bar{X}_{12}^{\prime} P_{\bar{G}_{2}} \bar{X}_{12}\right)^{-1}\end{array}\right]$.

\subsection{Asymptotic Distribution Theory}

This section studies asymptotic distributions of the PCIV estimators introduced in the last section. To derive the asymptotic distributions of the PCIV estimators $\hat{\delta}_{\text {PCIV }}$ and $\bar{\phi}_{\text {PCIV }}$, the following lemma will be used.

\section{LEMMA 2.}

(a) When $N_{j} \rightarrow \infty$, under Assumptions $1-4$ and 8 ,

$$
\sqrt{N_{j}}\left(\hat{\theta}_{j 1 \mathrm{IV}}-S_{j 1}^{\prime} \delta\right) \stackrel{d}{\rightarrow} N\left(0, \sigma_{u u} \operatorname{diag}\left(\frac{1}{\xi_{j 1}}, \ldots, \frac{1}{\xi_{j k_{1}}}\right)\right)=N\left(0, \sigma_{u u} V_{\delta j}\right), \text { say },
$$

where $\xi_{j 1}, \ldots, \xi_{j k_{1}}$ are $k_{1}$ largest eigenvalues of the matrix $M_{W_{j} G_{j}} M_{G_{j} G_{j}}^{-1} M_{G_{j} W_{j}}=$ $\operatorname{plim}_{N_{j} \rightarrow \infty}\left(1 / N_{j}\right) W_{j}^{\prime} P_{G_{j}} W_{j}$.

(b) When $N_{j} \rightarrow \infty$, under Assumptions 1, 3, 5, 6, and 8,

$$
\begin{aligned}
& \sqrt{N_{j}}\left(\bar{\tau}_{j 1 \mathrm{IV}}-\bar{S}_{j 1}^{\prime} \phi\right) \stackrel{d}{\rightarrow} N\left(0, \sigma_{u u} \operatorname{diag}\left(\frac{1}{\bar{\xi}_{j 1}}, \ldots, \frac{1}{\bar{\xi}_{j\left(k_{1}+k_{2}\right)}}\right)\right)=N\left(0, \sigma_{u u} \bar{V}_{\phi j}\right), \text { say, } \\
& \text { where } \bar{\xi}_{j 1}, \ldots, \bar{\xi}_{j\left(k_{1}+k_{2}\right)} \text { are } k_{1}+k_{2} \text { largest eigenvalues of the matrix } \\
& M_{\bar{W}_{j} \bar{G}_{j}} M_{\bar{G}_{j} \bar{G}_{j}} M_{\bar{G}_{j} \bar{W}_{j}}=\operatorname{plim}_{N_{j} \rightarrow \infty}\left(1 / N_{j}\right) \bar{W}_{j}^{\prime} P_{\bar{G}_{j}} \bar{W}_{j} .
\end{aligned}
$$

Remark. This lemma shows that $\hat{\theta}_{j 1 \mathrm{IV}}$ and $\bar{\tau}_{j 1 \mathrm{IV}}$ estimate particular linear combinations of the structural parameter vectors $\delta$ and $\phi$ consistently and that their 
asymptotic distributions are normal. Under the special case $S_{j 2}^{\prime} \delta=0$, the parameter vector $\delta$ can be estimated consistently by $S_{j 1} \hat{\theta}_{j 1 \mathrm{IV}}$, because

$S_{j 1} \hat{\theta}_{j 1 \mathrm{IV}}-S_{j 1} S_{j 1}^{\prime} \delta=S_{j 1} \hat{\theta}_{j 1 \mathrm{IV}}-\left(I-S_{j 2} S_{j 2}^{\prime}\right) \delta=S_{j 1} \hat{\theta}_{j 1 \mathrm{IV}}-\delta$.

No doubt, such a special case would rarely occur in practice. Likewise, when $\bar{S}_{j 2}^{\prime} \phi=0$, the parameter vector $\phi$ can be estimated consistently by $\bar{S}_{j 1} \bar{\tau}_{j 1 \mathrm{IV}}$.

Using Lemma 2 and formulae (24) and (26), the asymptotic distributions of the PCIV estimators for $\delta$ and $\phi$ can be obtained as follows.

\section{THEOREM 2.}

(a) Under Assumptions 1-4 and 8, as $N, N_{j} \rightarrow \infty(j=1,2)$,

$\sqrt{N}\left(\hat{\delta}_{\mathrm{PCIV}}-\delta\right) \stackrel{d}{\rightarrow} N\left(0, \sigma_{u u}\left(\Psi^{\prime} \Delta_{\delta} \Psi\right)^{-1}\right)$,

where $\Psi$ is the probability limit of $\Gamma, \Delta_{\delta}=\left[\begin{array}{cc}d_{1} V_{\delta 1}^{-1} & 0 \\ 0 & d_{2} V_{\delta 2}^{-1}\end{array}\right]$, and $d_{j}$ and $V_{\delta j}(j=$

1,2) are defined in Assumption 8 and Lemma 2, respectively.

(b) Under Assumptions 1, 3, 5, 6, and 8, as $N, N_{j} \rightarrow \infty(j=1,2)$,

$\sqrt{N}\left(\bar{\phi}_{\mathrm{PCIV}}-\phi\right) \stackrel{d}{\rightarrow} N\left(0, \sigma_{u u}\left(\bar{\Psi}^{\prime} \bar{\Delta}_{\phi} \bar{\Psi}\right)^{-1}\right)$,

where $\bar{\Psi}$ is the probability limit of $\bar{\Gamma}$ and $\bar{\Delta}_{\phi}=\left[\begin{array}{cc}d_{1} \bar{V}_{\phi 1}^{-1} & 0 \\ 0 & d_{2} \bar{V}_{\phi 2}^{-1}\end{array}\right]$ and $d_{j}$ and $\bar{V}_{\phi j}(j=$

1,2) are defined in Assumption 8 and Lemma 2, respectively.

Remarks.

(a) A simple calculation yields

$M_{W_{j} G_{j}} M_{G_{j} G_{j}}^{-1} M_{G_{j} W_{j}}=\left[\begin{array}{cc}\Pi_{1 j}^{\prime} M_{z_{1 j} z_{1 j}} \Pi_{1 j} & \Pi_{1 j}^{\prime} M_{z_{1 j} z_{1 j}} \\ M_{z_{1 j} z_{1 j}} \Pi_{1 j} & M_{z_{1 j} z_{1 j}}\end{array}\right]$.

Accordingly, the asymptotic variance-covariance matrix for $\hat{\delta}_{\text {PCIV }}$ depends only on $M_{z_{1 j} z_{1 j}}$ and $\Pi_{1 j}(j=1,2)$. In the same manner, the asymptotic variancecovariance matrix for $\bar{\phi}_{\text {PCIV }}$ depends only on $M_{z_{1 j} z_{1 j}}, M_{z_{1 j} z_{2 j}}, M_{z_{2 j} z_{2 j}}, \Pi_{1 j}$, and $\Pi_{2}$, because

$M_{\bar{W}_{j} \bar{G}_{j}} M_{\bar{G}_{j} \bar{G}_{j}}^{\bar{G}_{j_{j}}} M_{\bar{G}_{j} \bar{W}_{j}}=\left[\begin{array}{cc}\Pi^{j \prime} M_{\bar{z}_{j} \bar{z}_{j}} \Pi^{j} & \Pi^{j \prime} M_{\bar{z}_{j} \bar{z}_{j}} \\ M_{\bar{z}_{j} \bar{z}_{j}} \Pi^{j} & M_{\bar{z}_{j} \bar{z}_{j}}\end{array}\right]$,

where $\Pi^{j}=\left[\begin{array}{l}\Pi_{1 j} \\ \Pi_{2}\end{array}\right]$ and $M_{\bar{z}_{j} \bar{z}_{j}}=\left[\begin{array}{ll}M_{z_{1 j} z_{1 j}} & M_{z_{1 j} z_{2 j}} \\ M_{z_{2 j} z_{j j}} & M_{z_{2 j} z_{2 j}}\end{array}\right]$. It is interesting to observe that such limiting moments as $M_{h_{j} h_{j}}, M_{h_{j} z_{1 j}}, M_{f_{j} f_{j}}, M_{f_{j} z_{1 j}}$, and $M_{f_{j} z_{2 j}}$ do not affect the asymptotic variance-covariance matrices. This implies that the asymptotic efficiency of the PCIV estimators is not affected by the choice of instrument matrices $h_{i}$ and $f_{i}$ as long as they are uncorrelated with the disturbance term $u_{i}$. This counter-intuitive phenomenon occurs because the PCIV regression methods use only some largest eigenvalues of the projected sample moment matrix. 
(b) When $\Pi_{11}=\Pi_{12}$, we would have $M_{W_{1} G_{1}} M_{G_{1} G_{1}}^{-1} M_{G_{1} W_{1}} \simeq M_{W_{2} G_{2}} M_{G_{2} G_{2}}^{-1} M_{G_{2} W_{2}}$ because it is reasonable to expect that $M_{z_{11} z_{11}} \simeq M_{z_{12} z_{12}}$. Therefore, the matrix $\Psi^{\prime} \Psi$ is nearly singular, and the variance-covariance matrix in part (a) of Theorem 2 is not well defined. This also holds for part (b) of Theorem 2 under $\Pi_{11}=\Pi_{12}$.

(c) Now, assume the polar opposite case of Assumption 4 for the regression equation (1), that is, the case of $\Pi_{11}=\Pi_{12}=0$. Then, because $S_{j 11}^{\prime}=0$ where $S_{j 1}^{\prime}=$ $\left[\begin{array}{ll}S_{j 11}^{\prime n} & S_{j 12}^{\prime k_{1}}\end{array}\right]$, it is deduced from equation (23)

$\hat{\theta}_{j 1 \mathrm{IV}} \stackrel{p}{\rightarrow} \operatorname{plim}_{N_{j} \rightarrow \infty} S_{j 12}^{\prime} \gamma$.

This implies that $\hat{\theta}_{j 1 \mathrm{IV}}$ does not contain any information on the parameter vector $\beta$ and that $\beta$ cannot be estimated consistently. But the parameter vector $\gamma$ can be estimated consistently ${ }^{12}$ once we change the matrix $\Gamma$ such that $\Gamma=\left[\begin{array}{l}S_{112}^{\prime} \\ S_{212}^{\prime}\end{array}\right]$. The asymptotic distribution of the PCIV estimator for $\gamma$ can be recovered from Theorem 2. Intuitively speaking, the instruments we have chosen have no correlation with the regressor $y_{2 i}$ under $\Pi_{11}=\Pi_{12}=0$, and hence they cannot be valid instruments for $y_{2 i}$, which results in inconsistent estimation for $\beta$. In a more general case where $\left[\Pi_{11}^{\prime}, \Pi_{12}^{\prime}\right]^{\prime}$ is of deficient rank, we may also find by rotating the matrix $\left[\Pi_{11}^{\prime}, \Pi_{12}^{\prime}\right]^{\prime}$ as in Phillips (1989) and extending the analysis given earlier that $\beta$ cannot be estimated consistently but $\gamma$ can be. ${ }^{13}$

(d) When the polar opposite case of Assumption 4 for equation (10) holds (i.e., $\left.\Pi_{11}=\Pi_{12}=0\right)$ and when $\Pi_{2}=0$, the first $n$ columns of $\bar{S}_{j 11}^{\prime}$ are null vectors, and it follows as for equation (1) that $\beta$ cannot be estimated consistently but that $\gamma$ and $\rho$ can be. We may show that this also holds when $\left[\Pi_{11}^{\prime}, \Pi_{12}^{\prime}\right]^{\prime}$ is of deficient column rank and $\Pi_{2}=0$. In another polar case where $\Pi_{11}=\Pi_{12}=0$ and $\operatorname{rank}\left(\Pi_{2}\right)=n, M_{\bar{W}_{1} \bar{G}_{1}} M_{\bar{G}_{1} \bar{G}_{1}}^{-1} M_{\bar{G}_{1} \bar{W}_{1}} \simeq M_{\bar{W}_{2} \bar{G}_{2}} M_{\bar{G}_{2} \bar{G}_{2}} M_{\bar{G}_{2} \bar{W}_{2}}$ because it is reasonable to expect that $M_{\bar{z}_{1} \bar{z}_{1}} \simeq M_{\bar{z}_{2} \bar{z}_{2}}$. Therefore, $\bar{\Psi}^{\prime} \bar{\Psi}$ becomes nearly singular, and the asymptotic variance-covariance matrix of $\sqrt{N}\left(\bar{\phi}_{P C I V}-\phi\right)$ is not well defined. This can be shown to hold in a more general case where $\left[\Pi_{11}^{\prime}, \Pi_{12}^{\prime}\right]^{\prime}$ is of deficient rank and $\operatorname{rank}\left(\Pi_{2}\right)=n$.

(e) It would be interesting to specialize on the case $n=k_{1}=1$ for the regression equation (1), because this simple case would explicitly reveal the nonlinear influence of $\Pi_{1 j}$ and $M_{z_{1 j} z_{1 j}}$ on the asymptotic variances of $\hat{\beta}_{\text {PCIV }}$ and $\hat{\gamma}_{\mathrm{PCIV}}$. Using relation (27), the nonzero eigenvalue of $M_{W_{j} G_{j}} M_{G_{j} G_{j}}^{-1} M_{G_{j} W_{j}}$ and the corresponding eigenvector are obtained as

$\xi_{j 1}=M_{z_{1 j} z_{1 j}}+\Pi_{1 j}^{2} M_{z_{1 j} z_{1 j}}, \quad \operatorname{plim}_{N_{j} \rightarrow \infty} S_{j 1}=\left[\begin{array}{c}\frac{\Pi_{1 j}}{\sqrt{\Pi_{1 j}^{2}+1}} \\ \frac{1}{\sqrt{\Pi_{1 j}^{2}+1}}\end{array}\right]$.

Using these and part (a) of Theorem 2, we find that the asymptotic variancecovariance matrix of $\hat{\delta}_{\mathrm{PCIV}}$ is equivalent to that of the MD estimator given in (19). Thus, the same interpretations as in Section 3.2 can be given here. 


\section{MONTE CARLO SIMULATION}

This section reports the empirical biases, standard deviations, and root mean squared errors of the MD and PCIV estimators. Data for our experiments were generated according to

$$
\begin{aligned}
y_{1 i} & =\beta y_{2 i}+\gamma z_{1 i}+u_{i}, \quad \beta=\gamma=1(\text { for equation }(1)) ; \\
y_{1 i} & =\beta y_{2 i}+\gamma z_{1 i}+\rho z_{2 i}+u_{i}, \quad \beta=\gamma=\rho=1(\text { for equation }(10)) ; \\
y_{2 i} & =\Pi_{11} z_{1 i}+v_{2 i} \quad\left(i=1, \ldots, N_{1}\right) ; \Pi_{11}=1 ; \\
y_{2 i} & =\Pi_{12} z_{1 i}+v_{2 i} \quad\left(i=N_{1}+1, \ldots, N_{2}\right) ; \\
z_{1 i} & \sim i i d\left(\chi_{1}^{2}-1\right) ; z_{2 i} \sim i i d\left(\chi_{1}^{2}-1\right) ; \\
{\left[\begin{array}{c}
u_{i} \\
v_{2 i}
\end{array}\right] } & \sim i i d N\left(0,\left[\begin{array}{cc}
1 & \sigma_{u v} \\
\sigma_{u v} & 1
\end{array}\right]\right),
\end{aligned}
$$

where $z_{1 i}$ and $z_{2 i}$ were fixed throughout iterations and the number of iterations was set at 20,000. The PCIV estimator used $\left[z_{1 i}^{2}, z_{1 i}\right]$ as instruments for the seemingly unidentified structural equation under the rank condition failure and $\left[z_{1 i}^{2}, z_{1 i}, z_{2 i}\right]$ for the seemingly unidentified structural equation under the order condition failure.

First, we considered the case where the structural change points are known and utilized correctly in estimation. The experimental results for this case are reported in Table 1. Part I of Table 1 considers the seemingly unidentified structural equation under the rank condition failure (equation (1)) and part II that under the order condition failure (equation (10)). The experimental results in Table 1 can be summarized as follows.

- Part I shows that the PCIV estimator tends to have less standard deviation and root mean squared error than the MD estimator at $\left(N_{1}, N_{2}\right)=(50,50)$. But at $\left(N_{1}, N_{2}\right)=$ $(100,100)$, the converse is true. Regarding the bias, it is not certain which estimator performs better at $\left(N_{1}, N_{2}\right)=(50,50)$. But at $\left(N_{1}, N_{2}\right)=(100,100)$, the MD estimator tends to be less biased than the PCIV estimator.

- According to Part II, the PCIV estimator is less biased than the MD estimator excepting the case $\left(N_{1}, N_{2}\right)=(50,50), \sigma_{u v}=.5$ and $\Pi_{12} / \Pi_{11}=.25$. Furthermore, the PCIV estimator has less standard deviation and root mean squared error than the MD estimator in all cases.

- As the value of $\Pi_{12} / \Pi_{11}$ increases, the absolute value of bias, standard deviations, and root mean squared errors tend to decrease though there are a few exceptions.

- The effect of $\sigma_{u v}$ on bias, standard deviations, and root mean squared errors appears to be uncertain. But as the value of $\sigma_{u v}$ increases, the absolute value of bias tends to increase, though there are a few exceptions at $\left(N_{1}, N_{2}\right)=(50,50)$.

In practice, known structural change points may not be the true ones. Table 2 reports simulation results that use misspecified structural change points. The misspecified structural change points were selected randomly from intervals $[41,50]$ and $[52,61]$ at $\left(N_{1}, N_{2}\right)=(50,50)$ and from intervals $[81,100]$ and 
TABLE 1. Biases, standard deviations, and root mean square errors with known change points

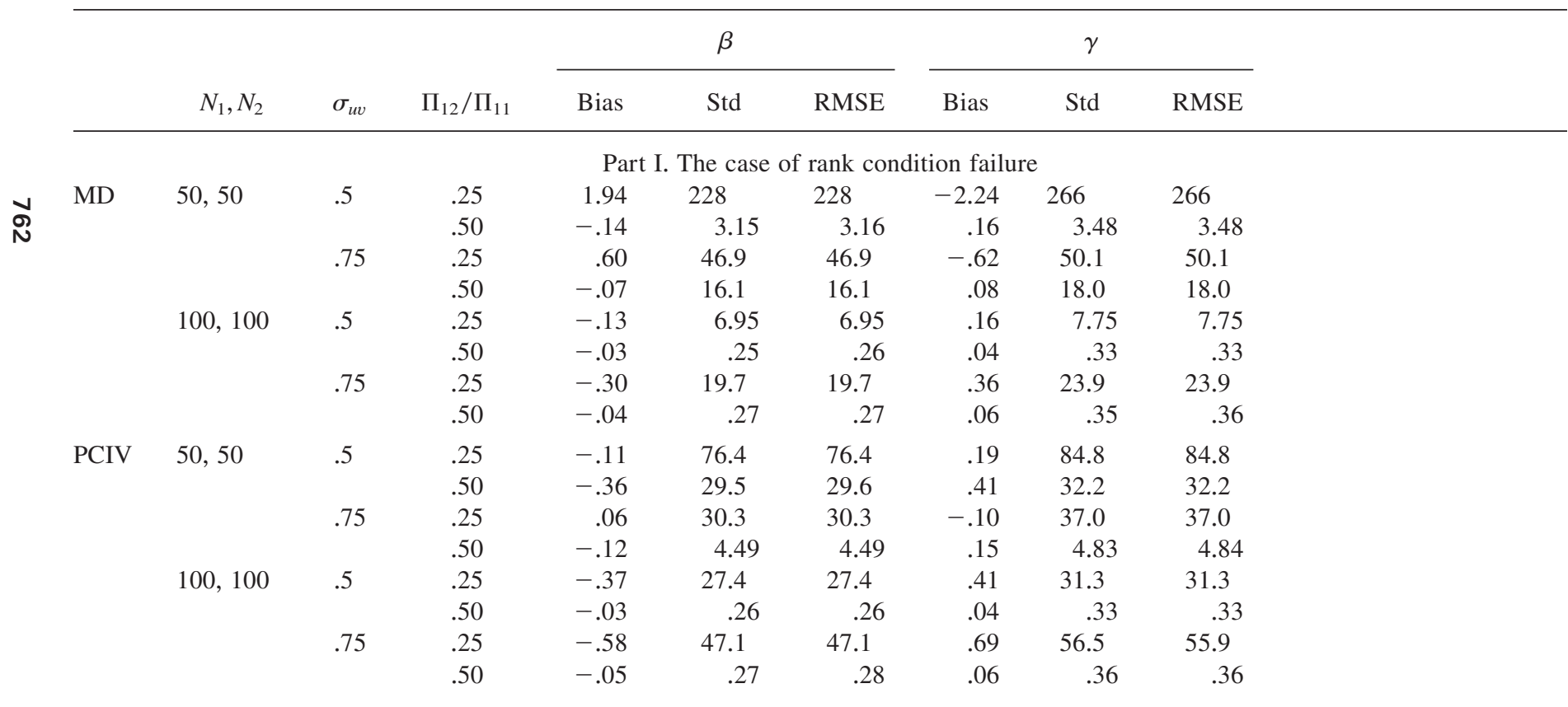




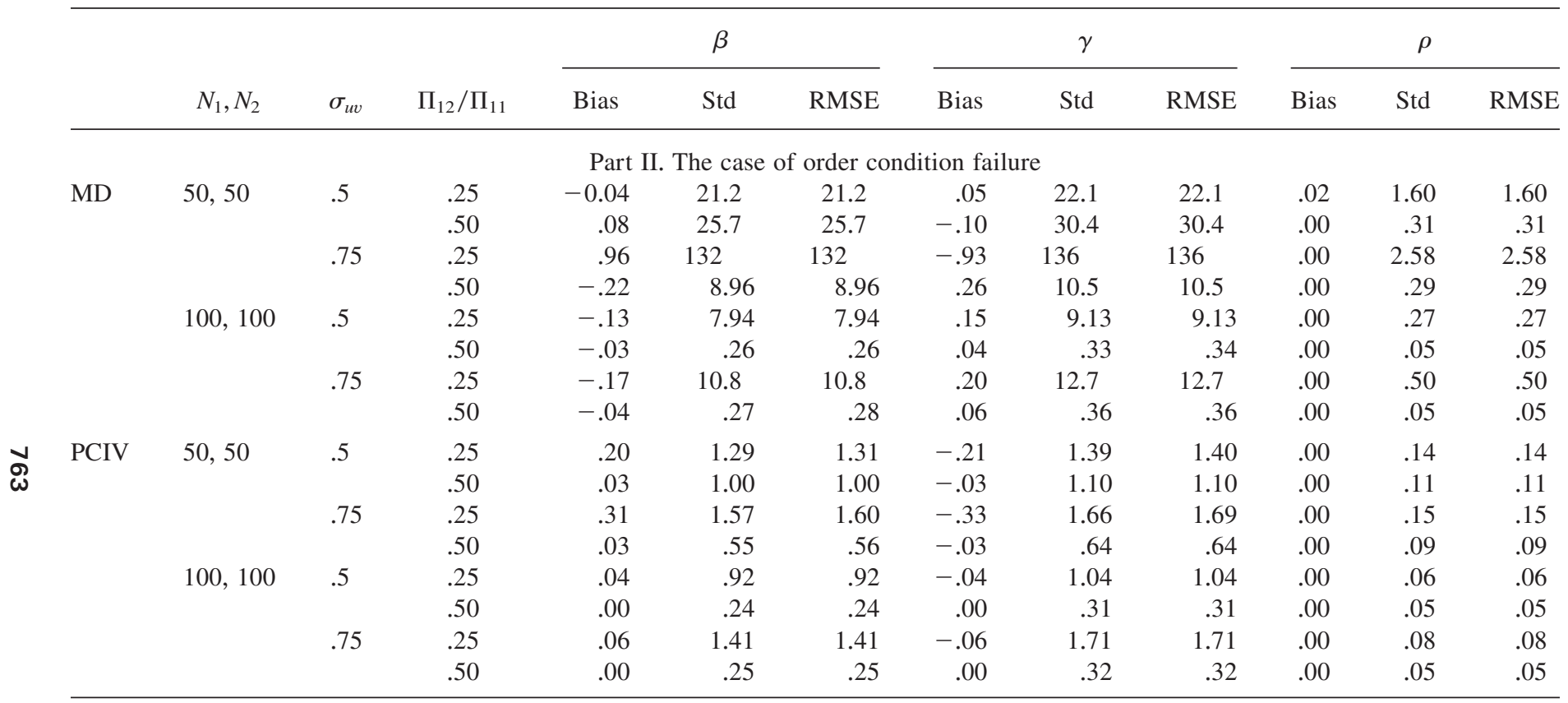

Note: Calculations are based on 20,000 iterations; $\Pi_{11}=1$. 
TABLE 2. Biases, standard deviations, and root mean square errors with misspecified change points

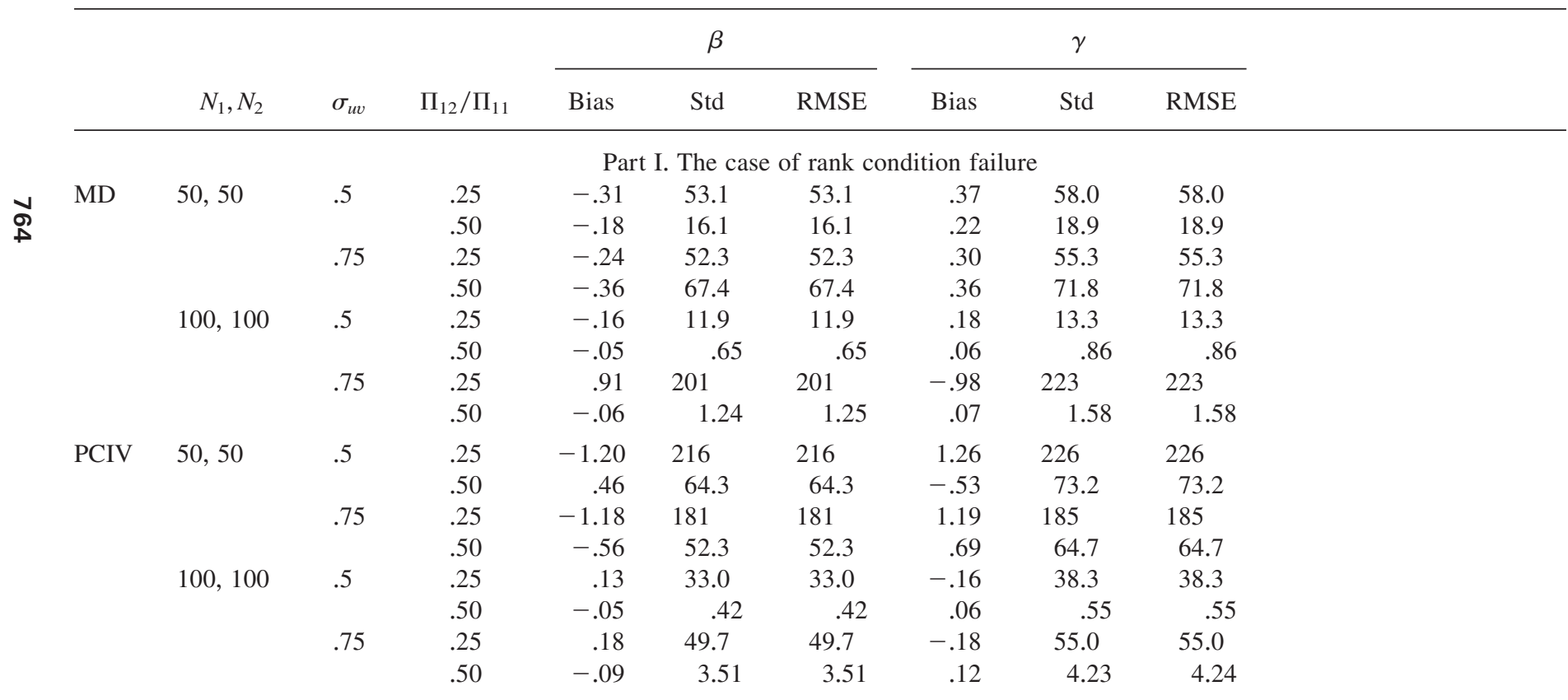




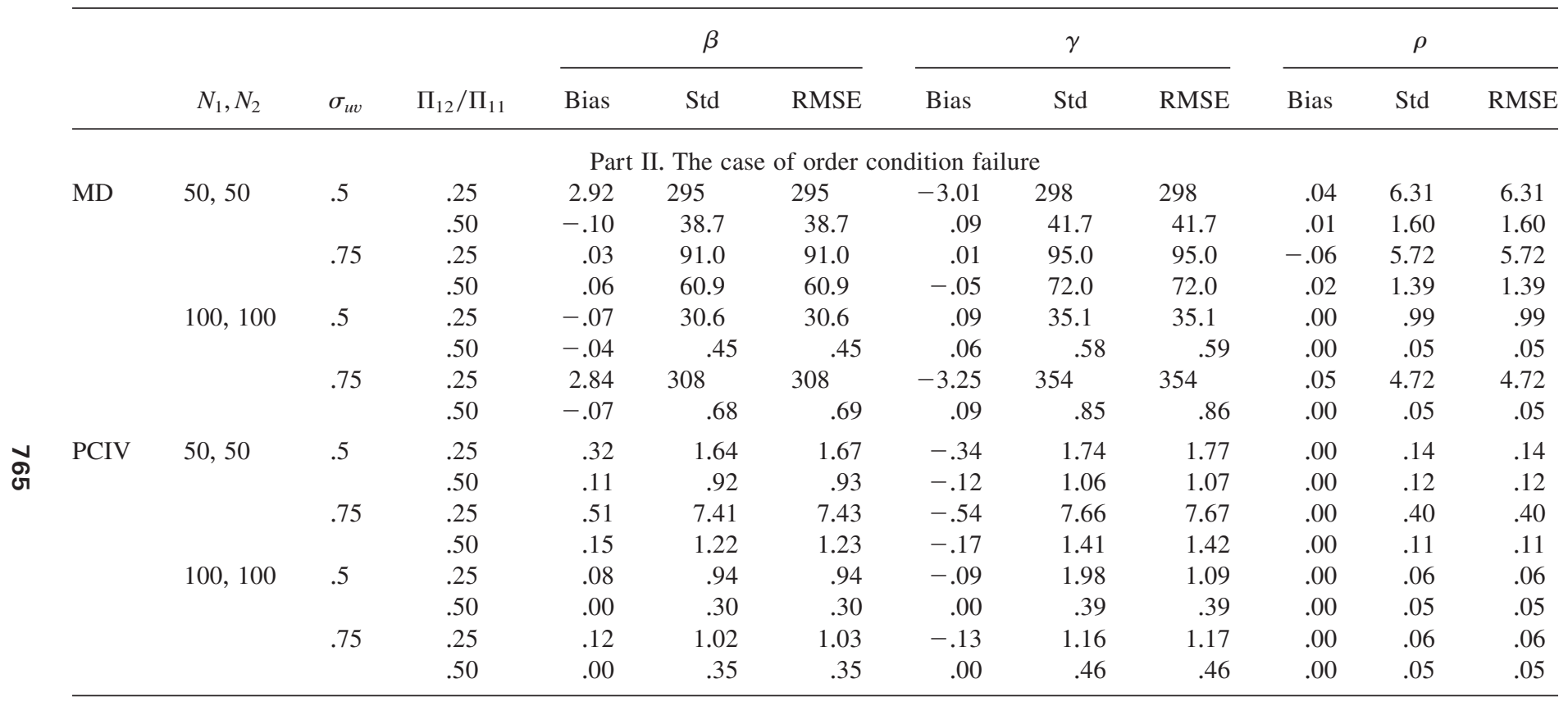

Notes: Calculations are based on 20,000 iterations; $\Pi_{11}=1$. The misspecified structural change points were selected randomly from intervals $[41,50]$ and $[52,61]$ at $N_{1}=N_{2}=50$ and from intervals $[81,100]$ and $[102,121]$ at $N_{1}=N_{2}=100$. 
TABLE 3. Biases, standard deviations, and root mean square errors with estimated change points

\begin{tabular}{|c|c|c|c|c|c|c|c|c|c|}
\hline & \multirow[b]{2}{*}{$N_{1}, N_{2}$} & \multirow[b]{2}{*}{$\sigma_{u v}$} & \multirow[b]{2}{*}{$\Pi_{12} / \Pi_{11}$} & \multicolumn{3}{|c|}{$\beta$} & \multicolumn{3}{|c|}{$\gamma$} \\
\hline & & & & Bias & Std & RMSE & Bias & Std & RMSE \\
\hline \multirow[t]{6}{*}{$\mathrm{MD}$} & 50,50 & .5 & .25 & .28 & .68 & .73 & -.30 & .73 & .79 \\
\hline & & & .50 & .07 & .43 & .43 & -.08 & .49 & .50 \\
\hline & & .75 & .25 & .42 & .64 & .77 & -.45 & .69 & .82 \\
\hline & & & .50 & .01 & .23 & .23 & -.02 & .30 & .30 \\
\hline & & .75 & .25 & .10 & .61 & .62 & -.11 & .70 & .71 \\
\hline & & & .50 & .02 & .24 & .24 & -.03 & .31 & .31 \\
\hline \multirow[t]{4}{*}{ PCIV } & 50,50 & .5 & .25 & .24 & 10.6 & 10.6 & -.26 & 12.2 & 12.2 \\
\hline & & & .50 & -.06 & 8.08 & 8.08 & .07 & 8.49 & 8.49 \\
\hline & & .75 & .25 & .39 & 15.1 & 15.1 & -.41 & 16.4 & 16.4 \\
\hline & & & .50 & .00 & .25 & .25 & .00 & .32 & .32 \\
\hline
\end{tabular}




\begin{tabular}{|c|c|c|c|c|c|c|c|c|c|c|c|c|}
\hline & \multirow[b]{2}{*}{$N_{1}, N_{2}$} & \multirow[b]{2}{*}{$\sigma_{u v}$} & \multirow[b]{2}{*}{$\Pi_{12} / \Pi_{11}$} & \multicolumn{3}{|c|}{$\beta$} & \multicolumn{3}{|c|}{$\gamma$} & \multicolumn{3}{|c|}{$\rho$} \\
\hline & & & & Bias & Std & RMSE & Bias & Std & RMSE & Bias & Std & RMSE \\
\hline MD & 50,50 & .5 & .25 & .22 & 4.24 & 4.24 & -.23 & 4.22 & 4.22 & .00 & .45 & .45 \\
\hline & & & .50 & .07 & .49 & .49 & -.08 & .55 & .56 & .00 & .09 & .09 \\
\hline & & .75 & .25 & .45 & 3.80 & 3.83 & -.47 & 3.59 & 3.63 & .00 & .25 & .25 \\
\hline & & & .50 & .01 & .23 & .23 & -.02 & .30 & .30 & .00 & .05 & .05 \\
\hline & & .75 & .25 & .09 & .73 & .73 & -.11 & .84 & .85 & .00 & .05 & .05 \\
\hline & & & .50 & .02 & .24 & .24 & -.03 & .31 & .31 & .00 & .05 & .05 \\
\hline \multirow[t]{4}{*}{ PCIV } & 50,50 & .5 & .25 & .33 & .85 & .91 & -.35 & .94 & 1.00 & .00 & .01 & .01 \\
\hline & & & .50 & .12 & .53 & .54 & -.13 & .61 & .63 & .00 & .09 & .09 \\
\hline & & .75 & .25 & .54 & 7.33 & 7.35 & -.57 & 7.57 & 7.59 & .00 & .39 & .39 \\
\hline & & & .50 & .04 & .23 & .23 & -.05 & .30 & .30 & .00 & .04 & .04 \\
\hline
\end{tabular}

Notes: Calculations are based on 20,000 iterations; $\Pi_{11}=1$. The structural change point was estimated by the least squares method, assuming there is only one structural change. 
$[102,121]$ at $\left(N_{1}, N_{2}\right)=(100,100)$. Note that the true change points are 51 and 101 at $\left(N_{1}, N_{2}\right)=(50,50)$ and $\left(N_{1}, N_{2}\right)=(100,100)$, respectively. The simulation results can be summarized as follows.

- As well expected, the estimators perform worse than those using correct change points, though there is an exception for the MD estimator at $\left(N_{1}, N_{2}\right)=(50,50)$.

- Part I shows that the MD estimator tends to perform better than the PCIV estimator at $\left(N_{1}, N_{2}\right)=(50,50)$ in terms of both bias and standard deviation. At $\left(N_{1}, N_{2}\right)=$ $(100,100)$, though, it seems difficult to decide which estimator performs better.

- According to Part II, the PCIV estimator performs uniformly better than the MD estimator in terms of standard deviation and root mean squared errors. The PCIV estimator also tends to be less biased.

The structural change points may not be known and need to be estimated in practice. Table 3 reports simulation results that use estimated structural change points. Assuming that there is only one structural change, the structural change points were estimated by the least squares method. ${ }^{14}$ The results can be summarized as follows.

- Tables 1 and 3 show that bias, standard deviations, and root mean squared errors tend to improve when estimated structural change points are used. For the MD estimator, this may be explained by the fact that the objective function for the least squares estimation of structural change point is related to the variance-covariance estimator of the MD estimator. For the PCIV estimator, it is less obvious why the estimated structural change points bring better results.

- Comparing the MD and PCIV estimators, the MD estimator tends to have less standard deviation and root mean squared error than the PCIV estimator in Part I, but the converse is true in Part II. Regarding the bias, the PCIV estimator tends to have less bias in Part I, but the converse holds in Part II.

- When $\left(N_{1}, N_{2}\right)=(100,100)$ and $\Pi_{12} / \Pi_{11}=.5$, the estimated structural change points are close to the true ones, and therefore the performance of the MD and PCIV estimator in Table 3 is similar to that in Table 1.

\section{AN EMPIRICAL EXAMPLE}

This section studies the Japanese export-GDP relation by using the methods proposed in previous sections and the Japanese deseasonalized quarterly data covering the period 1960:Q2-1998:Q3. Most previous time series studies investigating the export-GDP relation use the VAR causality tests (e.g., Shan and Sun, 1999; Marin, 1992; Ghartey, 1993). However, the VAR causality tests ignore contemporaneous relations which seem quite important in our empirical studies of Japanese exports and GDP, as will be seen shortly.

Instead of the VAR causality tests, we consider using the simultaneous equations system

$$
\begin{aligned}
& G D P R_{i}=\beta E X P R_{i}+\gamma^{\prime}\left(\begin{array}{c}
1 \\
E X P R_{i-1}
\end{array}\right)+\rho G D P R_{i-1}+u_{i}, \\
& E X P R_{i}=\Pi_{1}^{\prime}\left(\begin{array}{c}
1 \\
E X P R_{i-1}
\end{array}\right)+v_{2 i},
\end{aligned}
$$


where $G D P R_{i}$ denotes the GDP growth rate and $E X P R_{i}$ the export growth rate. ${ }^{15}$ Equation (28) signifies the structural equation and equation (29) the reduced form equation for $E X P R_{i}$. Equations (28) and (29) do not satisfy the order condition for identification and hence cannot be estimated by conventional methods. The Japanese quarterly data seem to support the AR(1) specification in equation (29). Notably, the lagged GDP growth rates do not seem to affect the current export growth rate. In equation (28), it may be more appropriate to include the variable $G D P R_{i-2} \cdot{ }^{16}$ But the results using the extra variable indicate that the coefficients for the export variables are not significant, which is counterintuitive. So we will focus only on equation (28).

The least squares methods for detecting structural change points (cf. Bai and Perron, 1998) find that there is a structural change in the intercept term of equation (29) at the period 1982:Q2. Moreover, the sup-Wald test of Andrews (1993) also indicates ${ }^{17}$ that there is one significant structural change in the intercept term of equation (29). Indeed, the average quarterly export growth rate before 1982:Q2 was $3.6 \%$, whereas that since 1982 :Q2 was only $0.7 \%$. Thus, we conclude that the Japanese export growth rate underwent a structural change during the sample period.

Now, assuming that the structural parameters were constant during the sample period, ${ }^{18}$ we estimated the coefficients of equation (28) by the MD and PCIV methods. The estimation results are reported in Table 4. As nonlinear instruments, $E X P R_{i-1}^{2}, E X P R_{i-1}^{3}$, and $\left(G D P R_{i-1}^{2}, E X P R_{i-1}^{2}\right)$ were used for the PCIV estimator. The structural change point was set at 1982:Q2.

The results in Table 4 indicate that all the regressors are significant at conventional significance levels. Because the averages of $G D P R_{i}$ and $G D P R_{i-1}$ and those of $E X P R_{i}$ and $E X P R_{i-1}$ are approximately the same, respectively, and because the intercept term is statistically insignificant, the estimation results from the MD estimation yield a relation

$\operatorname{Avg}(G D P R) \cong 0.583 \operatorname{Avg}(E X P R)-0.223 \operatorname{Avg}(E X P R)+0.371 A v g(G D P R)$

TABLE 4. PCIV estimation results for the Japanese export-GDP relation

\begin{tabular}{lcccc}
\hline Estimator & $E X P R$ & $\operatorname{EXPR}(-1)$ & $G D P R(-1)$ & $\operatorname{Avg}(G D P R) / \operatorname{Avg}(E X P R)$ \\
\hline MD & $.583\left(2.11^{*}\right)$ & $-.223(-1.84 *)$ & $.371\left(2.76^{* *}\right)$ & .57 \\
PCIV1 & $.636\left(3.00^{* *}\right)$ & $-.246(-2.39 *)$ & $.362(2.74 * *)$ & .61 \\
PCIV2 & $.630\left(3.04^{* *}\right)$ & $-.244(-2.39 *)$ & $.365\left(2.81^{* *}\right)$ & .61 \\
PCIV3 & $.407\left(3.48^{* *}\right)$ & $-.155\left(-2.48^{*}\right)$ & $.453\left(4.48^{* *}\right)$ & .46 \\
\hline
\end{tabular}

Notes: The numbers in parentheses are $t$-ratios. PCIV1, PCIV2, and PCIV3 use $E X P R_{i-1}^{2}, E X P R_{i-1}^{3}$, and $\left(E X P R_{i-1}^{2}, G D P R_{i-1}^{2}\right)$ as nonlinear instruments, respectively.

**: significant at the $1 \%$ level. *: significant at the $5 \%$ level. 
TABLE 5. OLS estimation results for the Japanese export-GDP relation

\begin{tabular}{lccc}
\hline $\operatorname{EXPR}$ & $\operatorname{EXPR}(-1)$ & $G D P R(-1)$ & $\operatorname{Avg}(G D P R) / \operatorname{Avg}(\operatorname{EXPR})$ \\
\hline $.722(3.67)$ & $-.113(-.54)$ & $.404(5.40)$ & 1.02 \\
\hline
\end{tabular}

Note: The numbers in parentheses are $t$-ratios.

or

$\operatorname{Avg}(G D P R) \cong \frac{0.583-0.223}{1-0.371} \operatorname{Avg}(\operatorname{EXPR})=0.57 \operatorname{Avg}(E X P R)$

where $\operatorname{Avg}(A)$ denotes the sample mean of $A_{i}$. We may infer from this relation that the $1 \%$ increase of export growth rate had the effect of increasing GDP by $0.57 \%$ on the average. Using the PCIV estimation results, we obtained similar numbers as reported in Table 4. In light of the fact that the average ratio of export to GDP was $11.2 \%$ during the sample period in Japan, relation (30) and the numbers in the last column of Table 4 seem to suggest that export had an accelerating multiplier effect on the GDP growth in Japan during the sample period.

The OLS estimation for equation (28) is subject to the problems of endogeneity and multicollinearity. To illustrate how serious these can be and to compare the results with those in Table 4, the OLS estimation was performed for equation (28). The results are reported in Table 5. Using the same arguments as before, we may interpret the results such that the $1 \%$ increase of export growth rate brings the $1.022 \%$ growth of GDP. This number seems to be too large relative to previous cross-sectional regression results in the literature (e.g., Lee and Cole, 1994). This must be due to the problems associated with estimating equation (28) by OLS.

\section{SUMMARY AND FURTHER REMARKS}

We have studied structural equations that do not satisfy the rank and/or order condition(s) for identification but still are identifiable. To estimate the seemingly unidentified structural equations, we proposed the MD and PCIV estimators. The asymptotic distributions of these estimators were reported. In a special case, these estimators were shown to have the same asymptotic distribution. But the asymptotic equivalence of these estimators in general could not be derived in this paper. Simulation results indicated that the MD and PCIV estimators were complementary to each other. But it was also found that the PCIV estimator tended to perform better in the case of order condition failure. The estimation methods proposed in this paper were applied to the Japanese export 
and GDP data. The results revealed that export had an accelerating multiplier effect on the GDP growth in Japan during the sample period 1960:Q2-1998:Q3.

There are a few topics that deserve further investigation but were not studied in this paper because of space limitations. First, the new identification conditions suggested in this paper are the rank conditions implying the presence of structural changes in the reduced form equation (Assumptions 4 and 6) and the absence of structural changes in the structural equation. The former condition can be tested by existing methods such as those of Gill and Lewbel (1992) and Cragg and Donald (1996), though these require modifications when structural change points are not known. But it seems that the latter condition cannot be tested by existing methods, and therefore new methods need to be developed. Second, this paper assumes that the structural change point is known. But estimating structural coefficients and unknown structural change points simultaneously would be a useful topic for future research. Third, this paper considers the case of identification failure in the conventional sense, but studying how structural changes affect the estimator properties in the case of weak instruments as in Staiger and Stock (1997) would be an interesting future research topic. Fourth, in light of prevalent evidence that many economic time series are nonstationary, it would be meaningful to study the seemingly unidentified structural equations under unit root nonstationarity.

\section{NOTES}

1. Hausman (1983), Hsiao (1983), Mariano (1982), and Phillips (1983) survey earlier contributions in this research area.

2. This estimator was kindly suggested by a referee.

3. For simplicity, we consider only two data regimes (or one structural change), but extension of the results in this paper to the case of several data regimes (or multiple structural changes) is straightforward.

4. Finite sample and asymptotic distribution theory of IV estimators under the assumption $\operatorname{rank}\left(\Pi_{2}\right)<n$, which covers Assumption 2 as a special case, is studied in Phillips (1989) and Choi and Phillips (1992). In addition, Staiger and Stock (1997) study the finite sample and asymptotic distribution theory of IV estimators when $\Pi_{2}=C / \sqrt{T}$ where $C$ is a constant matrix.

5. Testing the null hypothesis $H_{0}: \rho=0$ has attracted much attention from econometricians, and the tests for this null have been called overidentification tests (cf. Koopmans and Hood, 1953; Anderson and Kunitomo, 1992; Cragg and Donald, 1993).

6. See, for example, Newey and McFadden (1994).

7. See Judge and Bock (1983); Judge, Griffiths, Hill, Lütkepohl, and Lee (1985) and references therein.

8. When $n=k_{1}=1$, examples of instruments are $z_{1 i}^{2}, z_{1 i}^{4}$, and so on. Noninteger moments of $z_{1 i}$ can also be used as instruments when $z_{1 i}$ is nonnegative. When $z_{1 i}=\left[1, x_{1 i}^{\prime}\right]^{\prime}$, that is, when $z_{1 i}$ includes a constant term, we use $H\left(x_{1 i}\right)$ as instruments.

9. However, using the nonlinear functions of $z_{1 i}$ is not the main key to the estimability of unidentified structural equations. When other instruments are available and uncorrelated with the disturbance term $u_{i}$, they can also be used as instruments.

10. See the recent work by Lewbel (1997) and Dagenais and Dagenais (1995) and references therein.

11. Both $\hat{\theta}_{j 1 \mathrm{IV}}$ and $\hat{\delta}_{\mathrm{PCIV}}$ are called PCIV estimators here, about which the reader may be confused. But the former estimates a linear combination of the coefficient vector $\delta$, and the latter 
estimates the coefficient vector $\delta$ itself by using $\hat{\theta}_{11 \mathrm{IV}}$ and $\hat{\theta}_{21 \mathrm{IV}}$. Because there does not seem to be a danger of confusion, we will continue to use the term PCIV estimator to refer to both $\hat{\theta}_{j 1 \mathrm{IV}}$ and $\hat{\delta}_{\text {PCIV }}$.

12. Identifying relations (6) and (7) correspond with the facts that $\beta$ cannot be estimated consistently under $\Pi_{11}=\Pi_{12}=0$ and that $\gamma$ can be.

13. Consider the case $\left[\begin{array}{l}\Pi_{11} \\ \Pi_{12}\end{array}\right]=\left[\begin{array}{l}0 \\ \Upsilon\end{array}\right]$, where $\Upsilon$ is of full column rank. Then, $\Gamma=\left[\begin{array}{l}S_{11}^{\prime} \\ S_{12}^{\prime}\end{array}\right]=$ $\left[\begin{array}{ll}0 & P\end{array}\right]$ where $P$ is a nonzero matrix, and $\hat{\theta}_{j 1 \mathrm{IV}}$ do not contain any information on a certain part of $\beta$. Because $\Gamma$ is of deficient column rank in this case, $\hat{\delta}_{\text {PCIV }}$ is not well defined.

14. See, for example, Bai and Perron (1998) for the least squares estimation of structural change points in linear regression.

15. Because the GDP and export growth rates are log differences of GDP and export, respectively, these can be regarded as stationary.

16. The optimal VAR lag for $\left(G D P R_{i}, E X P R_{i}\right)^{\prime}$ is 2 according to the Bayesian information criterion.

17. The value of the sup-Wald test is 9.69 .

18. Existing structural change tests cannot be used for the structural equation (28) because the equation may not be estimable under some data regimes.

\section{REFERENCES}

Andrews, D.W.K. (1993) Tests for parameter instability and structural change with unknown change point. Econometrica 61, 821-856.

Anderson, T.W. \& N. Kunitomo (1992) Tests of overidentification and predeterminedness in simultaneous equation models. Journal of Econometrics 54, 49-78.

Angrist, J.D. \& A.B. Krueger (1995) Split sample instrumental variables estimates of the return to schooling. Journal of Business and Economic Statistics 13, 225-235.

Bai, J. \& P. Perron (1998) Estimating and testing linear models with multiple structural changes. Econometrica 66, 47-78.

Bekker, P. (1994) Alternative approximations to the distributions of instrumental variables estimator. Econometrica 62, 657-681.

Bekker, P. \& J. van der Ploeg (1999) Instrumental Variable Estimation Based on Grouped Data. Mimeo, Department of Economics, University of Groningen.

Bound, J., D.A. Jaeger, \& R.M. Baker (1995) Problems with instrumental variables estimation when the correlation between the instruments and the endogenous explanatory variable is weak. Journal of the American Statistical Association 90, 443-450.

Choi, I. \& P.C.B. Phillips (1992) Asymptotic and finite sample distribution theory for IV estimators and tests in partially identified structural equations. Journal of Econometrics 51, 113-150.

Choi, I. \& P.C.B. Phillips (1997) Regressions for Partially Identified, Cointegrated Simultaneous Equations. Cowles Foundation Discussion paper, Yale University.

Cragg, J.G. \& S.G. Donald (1993) Testing identifiability and specification in instrumental variables models. Econometric Theory 9, 222-240.

Cragg, J.G. \& S.G. Donald (1996) On the asymptotic properties of LDU-based tests of the rank of a matrix. Journal of the American Statistical Association 91, 1301-1309.

Dagenais, M.G. \& D.L. Dagenais (1995) Higher moment estimators for linear regression models with errors in the variables. Journal of Econometrics 76, 193-221.

Dufour, J.-M. (1997) Some impossibility theorems in econometrics with applications to structural and dynamic models. Econometrica 65, 1365-1388.

Ghartey, E.E. (1993) Causal relationship between exports and economic growth: Some empirical evidence in Taiwan, Japan and the US. Applied Economics 25, 1145-1152.

Gill, L. \& A. Lewbel (1992) Testing the rank and definiteness of estimated matrices with applications to factor, state-space, and ARMA models. Journal of the American Statistical Association 87, 766-776. 
Hausman, J. (1983) Specification and estimation of simultaneous equation models. In Z. Griliches \& M.D. Intriligator (eds.), Handbook of Econometrics, vol. 1. Amsterdam: North-Holland.

Hsiao, C. (1983) Identification. In Z. Griliches \& M.D. Intriligator (eds.), Handbook of Econometrics, vol. 1. Amsterdam: North-Holland.

Judge, G.G. \& M. Bock (1993) Biased estimation. In Z. Griliches \& M.D. Intriligator (eds.), Handbook of Econometrics, vol. 1. Amsterdam: North-Holland.

Judge, G.G., W.E. Griffiths, R.C. Hill, H. Lütekepohl, \& T.-S. Lee (1985) The Theory and Practice of Econometrics. New York: Wiley.

Koopmans, T.C. \& W.C. Hood (1953) The estimation of simultaneous linear economic relationships. In W.C. Hood \& T.C. Koopmans (eds.), Studies in Econometric Method, Cowles Foundation Monograph 10. New York: Wiley.

Lee, F.-Y. \& W.E. Cole (1994) Simultaneity in the study of exports and economic growth. International Economic Journal 8, 33-41.

Lewbel, A. (1997) Constructing instruments for regressions with mismeasurement error when no additional data are available with an application to patents and R\&D. Econometrica 65, 1201-1213.

Lucas, G. (1976) Econometric policy evaluation: A critique. In K. Brunner \& A.H. Meltzer (eds.), Carnegie-Rochester Conference Series on Public Policy, vol. 1. Amsterdam: North-Holland.

Mariano, R.S. (1982) Analytical small sample theory in econometrics: The simultaneous equations case. International Economic Review 23, 503-534.

Marin, D. (1992) Is the export-led growth hypothesis valid for industrialized countries? Review of Economics and Statistics 74, 678-688.

Muirhead, R. (1982) Aspects of Multivariate Statistical Theory. New York: Wiley.

Newey, W. (1990) Efficient instrumental variables estimation of nonlinear models. Econometrica 58, 809-837.

Newey, W. \& D. McFadden (1994) Large sample estimation and hypothesis testing. In R. Engle \& D.L. McFaddenr (eds.), Handbook of Econometrics, vol. 4. Amsterdam: North-Holland.

Perron, P. (1989) The great crash, the oil price shock, and the unit root hypothesis. Econometrica 57, 1361-1401.

Phillips, P.C.B. (1983) Exact small sample theory in the simultaneous equations model. In Z. Griliches \& M.D. Intriligator (eds.), Handbook of Econometrics, vol. 1. Amsterdam: North-Holland.

Phillips, P.C.B. (1989) Partially identified econometric models. Econometric Theory 5, 181-240.

Shan, J. \& F. Sun (1999) Export-led growth and the US economy: Some further testing. Applied Economics Letters 6, 169-172.

Sims, C.A. (1980) Macroeconomics and reality. Econometrica 48, 1-48.

Staiger, D. \& J.H. Stock (1997) Instrumental variables regression with weak instruments. Econometrica 65, 557-586.

Wang, J. \& E. Zivot (1998) Inference on structural parameters in instrumental variables regression with weak instruments. Econometrica 66, 1389-1404.

Zivot, E., Startz, \& Nelson (1998) Valid confidence intervals and inference in the presence of weak instruments. International Economic Review 39, 1119-1144.

\section{APPENDIX: PROOFS}

\section{Proof of Theorem 1.}

(a) Because $\hat{\pi}=\hat{\Pi} \delta+A U$, we may write $\hat{\delta}_{\mathrm{MD}}-\delta=\left(\hat{\Pi}^{\prime} \Upsilon_{\delta}^{-1} \hat{\Pi}\right)^{-1} \hat{\Pi}^{\prime} \Upsilon_{\delta}^{-1} A U$. 
But Assumption 7 yields $\hat{\Pi} \stackrel{p}{\rightarrow}\left[\begin{array}{ll}\Pi_{11} & I_{k} \\ \Pi_{12} & I_{k}\end{array}\right]$,

$$
\begin{aligned}
N Y_{\delta} & =N A A^{\prime}=\left[\begin{array}{cc}
\frac{N}{N_{1}}\left(\frac{1}{N_{1}} Z_{11}^{\prime} Z_{11}\right)^{-1} & 0 \\
0 & \frac{N}{N_{2}}\left(\frac{1}{N_{2}} Z_{12}^{\prime} Z_{12}\right)^{-1}
\end{array}\right] \\
& \stackrel{p}{\rightarrow}\left[\begin{array}{cc}
\frac{1}{d_{1}} M_{z_{11} z_{11}}^{-1} & 0 \\
0 & \frac{1}{d_{2}} M_{z_{12} z_{12}}^{-1}
\end{array}\right]
\end{aligned}
$$

and

$\sqrt{N} A U \stackrel{d}{\rightarrow} N\left(0, \sigma_{u u} \operatorname{plim}_{N \rightarrow \infty} N Y_{\delta}\right)$

from which the stated result follows.

(b) Using $\bar{\pi}=\bar{\Pi} \phi+B U$, write

$\bar{\phi}_{\mathrm{MD}}-\phi=\left(\bar{\Pi}^{\prime} \Upsilon_{\phi}^{-1} \bar{\Pi}\right)^{-1} \bar{\Pi}_{\phi}^{\prime} \Upsilon_{\phi}^{-1} B U$.

The result follows from the given assumptions, because $\bar{\Pi} \stackrel{p}{\rightarrow}\left[\begin{array}{ccc}\Pi_{11} & I_{k_{1}} & 0 \\ \Pi_{12} & I_{k_{1}} & 0 \\ \Pi_{2} & 0 & I_{k_{2}}\end{array}\right]$,

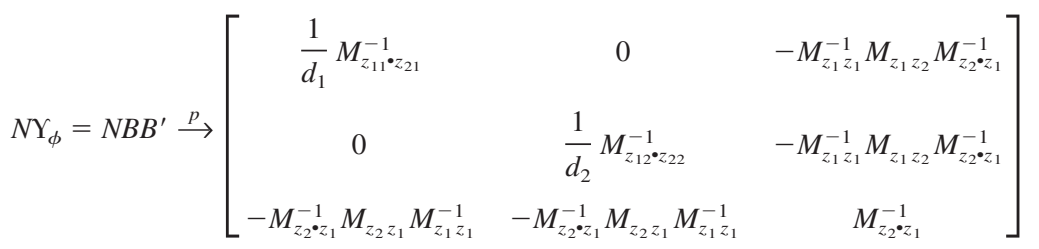

and

$\sqrt{N} B U \stackrel{d}{\rightarrow} N\left(0, \sigma_{u u} \operatorname{plim}_{N \rightarrow \infty} N \mathrm{Y}_{\phi}\right)$.

Note that part (b) of Assumption 7 implies that $M_{z_{11} *_{21}}$ and $M_{z_{12} z_{22}}$ are nonsingular (cf. Muirhead, 1982, p. 586). Moreover, because $(1 / N) Z^{\prime} Z=Z^{1 \prime} Z^{1}+Z^{2 \prime} Z^{2}$, the probability limit of $(1 / N) Z^{\prime} Z$ is a positive definite matrix by part (b) of Assumption 7. This implies that $M_{z_{1} z_{1}}$ and $M_{z_{2} \cdot z_{1}}$ are nonsingular.

\section{Proof of Lemma 1.}

(a) Let $H_{j}=\left[h_{i}\right]_{i \in \mathcal{G}_{j}}$. Because $\left(1 / N_{j}\right) V_{2 j}^{\prime} H_{j},\left(1 / N_{j}\right) V_{2 j}^{\prime} Z_{1 j} \stackrel{p}{\rightarrow} 0$ under Assumption 8 , it follows that

$$
\frac{1}{N_{j}} W_{j}^{\prime} G_{j} \stackrel{p}{\rightarrow}\left[\begin{array}{cc}
\Pi_{1 j}^{\prime} M_{z_{1 j} h_{j}} & \Pi_{1 j}^{\prime} M_{z_{1 j} z_{1 j}} \\
M_{z_{1 j} h_{j}} & M_{z_{1 j} z_{1 j}}
\end{array}\right]=M_{W_{j} G_{j}},
$$


which shows that $\operatorname{rank}\left(M_{W_{j} G_{j}}\right)=k_{1}$. Because $M_{G_{j} G_{j}}$ can be factorized as $M_{G_{j} G_{j}}=M_{G_{j} G_{j}}^{1 / 2} M_{G_{j} G_{j}}^{1 / 2}$ by Assumption 8, where $M_{G_{j} G_{j}}^{1 / 2}$ is of full rank, we have $\operatorname{rank}\left(M_{W_{j} G_{j}} M_{G_{j} G_{j}}^{-1 / 2}\right)=k_{1}$, which completes the proof.

(b) Using the same methods as for part (a) yields the required result.

LEMMA 3. Under Assumption 8,

(a) $\left(1 / N_{j}\right) \lambda_{j i} \stackrel{p}{\rightarrow} \xi_{j i}\left(i=1, \ldots, n+k_{1}\right)$, where $\xi_{j i}$ is as defined in Lemma 2 .

(b) $S_{j 1} \stackrel{p}{\rightarrow} \Psi_{j 1}$, where $\Psi_{j 1}$ is a matrix whose columns are eigenvectors of the matrix $M_{W_{j} G_{j}} M_{G_{j} G_{j}}^{-1} M_{G_{j} W_{j}}$ corresponding to $k_{1}$ largest eigenvalues.

\section{Proof.}

(a) $\lambda_{j i}$ satisfies the determinantal equation $\left|\left(1 / N_{j}\right) W_{j}^{\prime} P_{G_{j}} W_{j}-\left(1 / N_{j}\right) \lambda_{j i} I\right|=0$. But $\left(1 / N_{j}\right) W_{j}^{\prime} P_{G_{j}} W_{j} \stackrel{p}{\rightarrow} M_{W_{j} G_{j}} M_{G_{j} G_{j}}^{-1} M_{G_{j} W_{j}}$, from which the result follows.

(b) The matrix $S_{j 1}$ satisfies the equation $\left(1 / N_{j}\right) W_{j}^{\prime} P_{G_{j}} W_{j} S_{j 1}=\left(1 / N_{j}\right) \lambda_{j i} S_{j 1}$ and $\left(1 / N_{j}\right) W_{j}^{\prime} P_{G_{j}} W_{j} \stackrel{p}{\rightarrow} M_{W_{j} G_{j}} M_{G_{j} G_{j}}^{-1} M_{G_{j} W_{j}}$. Thus, we obtain the stated result by using part (a) of Lemma 3 .

\section{Proof of Lemma 2.}

(a) Because $S_{j 1}^{\prime} S_{j 2}=0$, it follows that $\hat{\theta}_{j 1 \mathrm{IV}}=S_{j 1}^{\prime} \delta+\left(X_{1 j}^{\prime} P_{G_{j}} X_{1 j}\right)^{-1} X_{1 j}^{\prime} P_{G_{j}} U_{j}$. But Lemma 3 gives

$$
\begin{aligned}
\frac{1}{N_{j}} X_{1 j}^{\prime} P_{G_{j}} X_{1 j} & =\frac{1}{N_{j}} S_{j 1}^{\prime} W_{j}^{\prime} G_{j}\left(G_{j}^{\prime} G_{j}\right)^{-1} G_{j}^{\prime} W_{j} S_{j 1} \\
& =\frac{1}{N_{j}} \operatorname{diag}\left(\lambda_{j 1}, \ldots, \lambda_{j k_{1}}\right) \stackrel{p}{\rightarrow} \operatorname{diag}\left(\xi_{1}, \ldots, \xi_{k_{1}}\right),
\end{aligned}
$$

and, as a result of Assumption 8,

$$
\frac{1}{\sqrt{N_{j}}} X_{1 j}^{\prime} P_{G_{j}} U_{j}=\frac{1}{\sqrt{N_{j}}} S_{j 1}^{\prime} W_{j}^{\prime} G_{j}\left(G_{j}^{\prime} G_{j}\right)^{-1} G_{j}^{\prime} U_{j} \stackrel{d}{\rightarrow} N\left(0, \sigma_{u u} \operatorname{diag}\left(\xi_{1}, \ldots, \xi_{k_{1}}\right)\right) .
$$

Relations (A.1) and (A.2) yield the stated result.

(b) This is obtained by using the same methods as for part (a).

Proof of Theorem 2. Choose a matrix $C_{j}$ such that $C_{j}^{\prime} C_{j}=N_{j} M_{W_{j} G_{j}} M_{G_{j} G_{j}}^{-1} M_{G_{j} W_{j}}$. At $N_{j}=\infty, \sqrt{N_{j}}\left(\hat{\theta}_{j 1 \mathrm{IV}}-S_{11}^{\prime} \delta\right)$ has the same distribution as $\left(C_{j}^{\prime} C_{j}\right)^{-1} C_{j}^{\prime} U_{j}$. But $E\left[\left(C_{1}^{\prime} C_{1}\right)^{-1} C_{1}^{\prime} U_{1} U_{2}^{\prime} C_{2}^{\prime}\left(C_{2}^{\prime} C_{2}\right)^{-1}\right]=0$ for any choice of the matrices $C_{1}$ and $C_{2}$. Therefore, $\sqrt{N_{1}}\left(\hat{\theta}_{11 \mathrm{IV}}-S_{11}^{\prime} \delta\right)$ and $\sqrt{N_{2}}\left(\hat{\theta}_{21 \mathrm{IV}}-S_{21}^{\prime} \delta\right)$ are independent at $N_{1}, N_{2}=\infty$. Now, writing

$$
\sqrt{N}\left(\hat{\delta}_{\mathrm{PCIV}}-\delta\right)=\left(\Gamma^{\prime} \Theta_{\delta}^{-1} \Gamma\right)^{-1} \Gamma^{\prime} \Theta_{\delta}^{-1}\left[\begin{array}{c}
\sqrt{N / N_{1}} \sqrt{N_{1}}\left(\hat{\theta}_{11 \mathrm{IV}}-S_{11}^{\prime} \delta\right) \\
\sqrt{N / N_{2}} \sqrt{N_{2}}\left(\hat{\theta}_{21 \mathrm{IV}}-S_{21}^{\prime} \delta\right)
\end{array}\right]
$$

and using part (a) of Lemma 2, part (b) of Lemma 3, and (A.1) completes the proof for part (a). Part (b) can be shown in a similar manner. 